\title{
Microstructural Response of SiIm and SBS Block Copolymers to Heat Treatment
}

\author{
Richard J. SPONTAK and Michael C. Williams ${ }^{\dagger}$ \\ Center for Advanced Materials, Lawrence Berkeley Laboratory, \\ Berkeley, CA 94720-9989, U.S.A., and \\ Department of Chemical Engineering, University of California, \\ Berkeley, CA 94720-9989, U.S.A.
}

(Received March 2, 1988)

\begin{abstract}
Using dynamic transmission electron microscopy, we have studied in-situ the effects of thermal curing/annealing on microstructural elements within films of unstained siloxaneimide (SiIm) and stained styrene-butadiene-styrene (SBS) block copolymers. Samples of the SiIm multiblock copolymer, only partially cured, were heated to $100^{\circ} \mathrm{C}$ and $212^{\circ} \mathrm{C}$, with the appearance of siloxane domains increasing with curing time. Microstructural coalescence was observed at $324^{\circ} \mathrm{C}$, suggesting the presence of a phase transition. SBS transition kinetics are prefaced by theoretical predictions of the equilibrium separation temperature $T_{\mathrm{s}}$ as a function of molecular weight and composition, leading to $T_{\mathrm{s}} \propto \bar{M}_{n}{ }^{0.8}$. Since the presence of a stain alters all kinetic phenomena, a model based on free-volume theory was devised to estimate how the stain concentration affects local viscosity. Annealing of initially non-equilibrated SBS sample films was observed to cause increased ordering of styrene domains as temperature increased, with phase inversion at elevated temperatures. Lamellar development was found to occur by coarsening in areas where some lamellae initially formed; structure bands, measuring $110 \mathrm{~nm}$ across and separated by $280 \mathrm{~nm}$, were observed to form in initially homogeneous regions.
\end{abstract}

KEY WORDS Block Copolymer / Dynamic TEM / Heat Treatment

Microphase Separation / Microstructural Evolution / Multiblock

Copolymer / Transmission Electron Microscopy /

Microphase separation in block copolymers, due to thermodynamic incompatibility between the blocks, results in microstructures, or domains, which are on the size scale of the end-to-end distance of the polymer molecules. These microstructures and their corresponding interphase regions are responsible for thermal and mechanical properties that are unlike those of either homopolymer or those of a random copolymer of equal composition. Direct studies of these microstructures are restricted by the small size of the domains (typically on the order of tens of nanometers); consequently, only some experimental methods-e.g., transmission electron micros- copy (TEM) ${ }^{1-4}$ small-angle X-ray scattering (SAXS),${ }^{5-7}$ and small-angle neutron scattering (SANS) ${ }^{8-10}$-have proven useful in accurately characterizing the microstructures of block copolymers.

A limited amount of experimental evidence has been reported, however, concerning thermally-induced microstructural transitions from a structured (phase-separated) to a homogeneous state (or vice-versa) in poly(styrene-butadiene) and poly(siloxane-imide) block copolymers. Initial light-transmission experiments by Leary and Williams ${ }^{11}$ indicated a phase transition, denoted as $T_{\mathrm{s}}$, wherein the well-developed microstructures

\footnotetext{
† To whom correspondences should be addressed.
} 
in styrene-butadiene-styrene (SBS) block copolymers disappeared at an elevated temperature. The presence of $T_{\mathrm{s}}$ has since been substantiated by investigators for SB, SBS, and styrene-isoprene star-branched $(\mathrm{SI})_{x}$ copolymers using the fact that the structure peak within $\mathrm{SAXS}^{4,5}$ and $\mathrm{SANS}^{8}$ intensity patterns diverges at the microphase-separation temperature. Indirect methods of determining $T_{\mathrm{s}}$ have also been established. Leung and Koberstein, ${ }^{12}$ for example, used differential scanning calorimetry (DSC) to demonstrate a $T_{\mathrm{s}}$ in polyether-based polyurethane block copolymers. In addition, by finding the temperature at which the storage $\left(G^{\prime}\right)$ and loss $\left(G^{\prime \prime}\right)$ moduli become insensitive to temperature, Han and $\mathrm{Kim}^{13}$ have used dynamic mechanical testing (DMT) to report $T_{\mathrm{s}}=230^{\circ} \mathrm{C}$ for an SIS block copolymer.

Although experimental evidence of $T_{\mathrm{s}}$ is still not voluminous, this transition is vital in accurately modeling the phase behavior of block copolymers. The original concept of $T_{\mathrm{s}}$ was postulated as a first-order transition by Leary and Williams, ${ }^{14}$ whose theory was based on free-energy minimization upon equilibration. Modifications of that theory, ${ }^{3,15}$ as well as development of other theories based upon the same energetics principle, have led to qualitatively correct predictions for $T_{\mathrm{s}}$. Recent works by Leibler, ${ }^{16}$ using a mean-field theory to describe the microphase transition, and by Fredrickson and Larson, ${ }^{17,18}$ considering the effects of critical density fluctuations upon phase separation, have prompted a renewed interest in this phenomenon, with current focus on the kinetics of phase separation. ${ }^{19}$

To obtain a better understanding of the formation and evolution of microstructures in microphase-separated block copolymers, we present here a method by which the thermal response of microstructures can be directly observed in-situ. Two very different types of copolymer are studied, one poly(siloxaneimide) multiblock and three poly(styrenebutadiene) triblock copolymers.

\section{EXPERIMENTAL}

\section{Materials}

The poly(siloxane-imide) (SiIm) copolymer, grade 3510 manufactured by M\&T Chemicals Inc. (Rahway, NJ 07065), is designated here MT3 for brevity and is characterized in Table I. The chemical structure of MT3 has not been fully revealed by the supplier. Thus, we present in Figure 1 the structure of a similar SiIm copolymer $^{20}$ in an attempt to clarify the general chemical nature of the copolymer, in which the hard block consists of a crystalline polyimide $\left(-\mathrm{NC}_{2} \mathrm{O}_{2} \mathrm{R}_{1} \mathrm{C}_{2} \mathrm{O}_{2} \mathrm{NR}_{4}-\right)$ and the soft block is composed of diamine $\left(-\mathrm{NC}_{2} \mathrm{O}_{2} \mathrm{R}_{1} \mathrm{C}_{2} \mathrm{O}_{2} \mathrm{NR}_{2}-\right)$ and siloxane segments. This particular copolymer is the result of a polycondensation reaction, ${ }^{21,22}$ in which siloxane blocks of the form $\left[-\mathrm{Si}-(\mathrm{R})_{2}-\mathrm{O}-\right]_{x}$,

Table I. Material properties of the block copolymers used in this study ${ }^{\mathrm{a}}$

\begin{tabular}{|c|c|c|c|c|c|}
\hline Sample ${ }^{b}$ & Architecture & Morphology & $\frac{\bar{M}_{n} \times 10^{-3}}{\mathrm{~g} \mathrm{~mol}^{-1}}$ & $\bar{M}_{w} / \bar{M}_{n}$ & $\frac{\text { Soft block }^{\mathrm{c}}}{\mathrm{wt} \%}$ \\
\hline MT3 & $(\mathrm{SiIm})_{n}$ & SPH & $50-100$ & $\approx 2.0$ & 74 \\
\hline F411 & SBS & CYL & 115.0 & 2.00 & 70 \\
\hline F414 & SBS & LAM & 87.7 & 1.71 & 60 \\
\hline F416 & SBS & CYL & 84.8 & 1.65 & 70 \\
\hline
\end{tabular}

a Characterized by the manufacturers.

b MT3 is an abbreviation for the 3510 grade Silm copolymer (M\&T Chemicals, Inc.), and F is an abbreviation for Finaprene SB/SBS copolymers (Cosden Oil and Chemical Co.) of various grades.

c This includes the siloxane and diamine segments for MT3 and only the butadiene block in the SBS copolymers. 


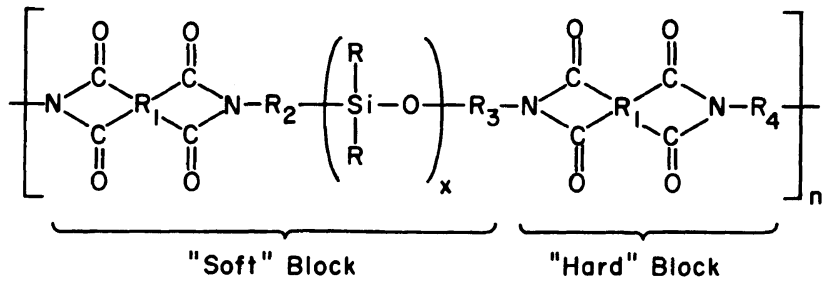

Figure 1. Chemical structure of an SiIm copolymer showing the "soft" block (the siloxane and diamine segments) and the "hard" imide block. Substituent groups denoted by $R$ (with subscripts $1-4$ or without subscript) are proprietary and, consequently, unknown.

where the identity of the $\mathbf{R}$ group is unknown, ${ }^{23}$ are incorporated directly into the backbone of a polyimide resin. ${ }^{24,25}$ The sample was received as a $25 \%$ solution in $N$ methyl-2-pyrrolidone (NMP), from which dilute solutions (needed for casting ultrathin films) were prepared using fresh NMP under dry nitrogen conditions. To obtain the desirable ultimate properties of this copolymer, the supplier ${ }^{25}$ recommends solvent removal by the following cure cycle: one hour at $100^{\circ} \mathrm{C}$, one hour at $150^{\circ} \mathrm{C}$, and one hour at $200^{\circ} \mathrm{C}$.

The styrene-butadiene copolymers are manufactured by Cosden Oil and Chemical Co. (Deer Park, TX 77536) and consist of three triblock (SBS) copolymers. These three copolymers are also characterized in Table I. Previous work ${ }^{2,3}$ with films of these copolymers, cast from dilute toluene solutions, reveal dispersed polystyrene (PS) domains in a polybutadiene (PB) matrix or co-continuous lamellar structure, depending on the bulk PS fraction.

\section{Sample Preparation}

Using low-voltage (conventional) TEM required films or sections thinner than $100 \mathrm{~nm}$. Preparation of ultrathin films, approximately $30-40 \mathrm{~nm}$ thick, ${ }^{2}$ was accomplished utilizing a direct-casting technique (presented elsewhere ${ }^{26}$ ). However, variations in this technique, required here to permit observation of dynamic thermally-induced microprocesses, warrant a full explanation of the direct-casting procedure. In the cases of both the SBS and

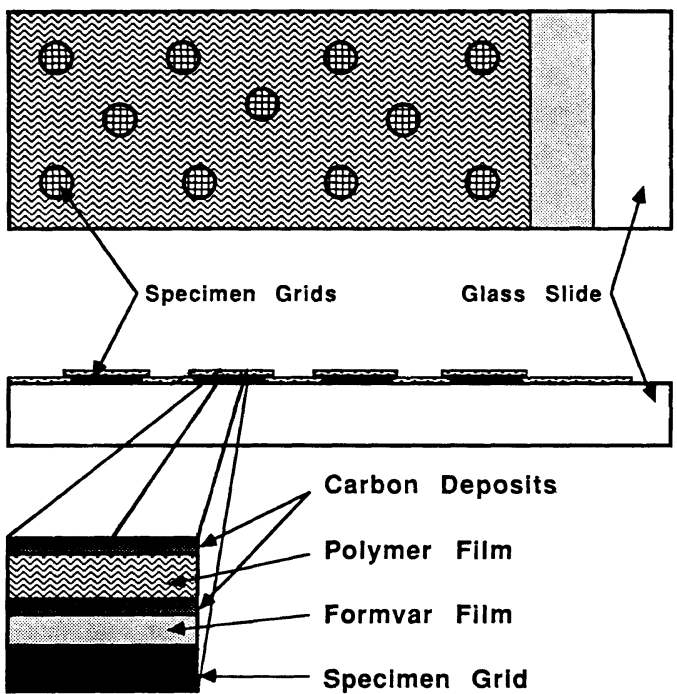

Figure 2. Schematic of the sample-preparation technique used to obtain stable ultrathin films $(30-40 \mathrm{~nm}$ thick $^{2}$ ). The overall figure shows the position of the copper grids on a clean glass slide. The enlarged section shows the layers of materials used. First, the Formvar support film is cast, and carbon is deposited atop the film to isolate the Formvar. The polymer film is cast over the carbon and is stained, if necessary. Another coat of carbon is added to the polymer film for stability.

Silm copolymers, dilute solutions (approximately $0.5 \%$ ) were prepared in toluene and NMP, respectively. To ensure singlephase behavior within the dilute SBS solutions, ${ }^{7,27,28}$ the solutions were heated to $90^{\circ} \mathrm{C}$ (the normal boiling point of toluene is 109. $\left.6^{\circ} \mathrm{C}\right)$.

Four-hundred-mesh copper grids, selected to provide ultrathin films with a high degree of structural support, were coated ${ }^{29}$ with a 
Formvar film cast from a $0.25 \%$ solution in dichloroethane. This film, adhering to the grids on a clean glass slide (Figure 2) and measuring approximately $10 \mathrm{~nm}$ thick, was then subjected to carbon vapor deposition in a Mikros-Varian evaporator (Varian Associates, Palo Alto, CA 92680) to isolate the Formvar from the surrounding environment. The ultrathin films were prepared by casting the dilute copolymer solutions directly atop the carbon coat by dipping the glass slide into the solutions. Instead of allowing the SBS films to dry slowly and thereby attain equilibrated microstructures (as in ref 26), we placed them directly into a vacuum desiccator. Solvent removal under house vacuum conditions for two days was considered almost complete for the SBS copolymer films. After being dried, the films were stained with the vapor of a $1.0 \%$ osmium tetroxide aqueous solution for $80 \mathrm{~min}$ to enhance contrast between the microphases. Under these stain conditions, the stain intensity is proportional to the density of double bonds present in the PB fraction. ${ }^{1,30}$ After staining, the films were again coated with vapor sputtered from carbon or carbonplatinum rods; the carbon was used here to protect the film and guarantee its stability (up to $45 \mathrm{~min}^{2,26}$ ) under the electron beam, whereas platinum was used, on occasion, to decorate the film surface.

Since only heating can fully remove solvent from the MT3 copolymer, ${ }^{25}$ NMP was still present in the uncured ultrathin films, despite the vacuum environment. Solvent was eliminated from some films by the full-cure heating cycle (see above), thus providing a reference state to display well-developed microstructures. Other films were partially cured according to the following cycle: $2 \mathrm{~h}$ at $85^{\circ} \mathrm{C}$ and $2 \mathrm{~h}$ at $120^{\circ} \mathrm{C}$. The upper temperature of this cycle was selected on the basis of the material's thermo-mechanical properties (the yield temperature, the temperature at which the material began to flow, was observed ${ }^{23}$ to be about $130^{\circ} \mathrm{C}$ ). Even with this partial-cure cy- cle, though, the film may still contain from 6 $22 \mathrm{wt} \% \mathrm{NMP}$, according to the work of Davis et al. ${ }^{31}$ with similar SiIm copolymers dissolved in NMP. Solvent removal during the dynamic TEM studies was then expected. To allow solvent evaporation within the microscope, the second carbon-vapor deposition (used in preparing SBS films) was omitted from the sample preparation; this afforded no stability problems since copolymers of this type are re-

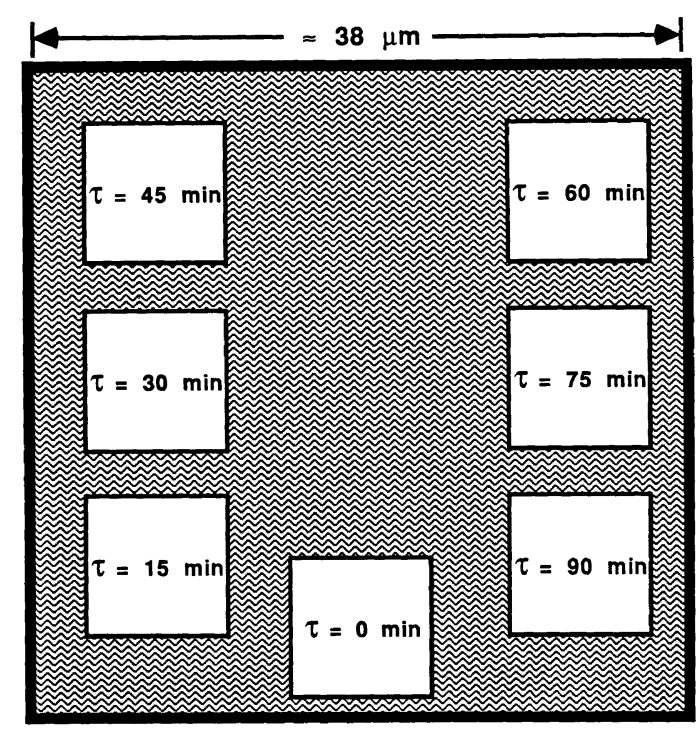

(a)

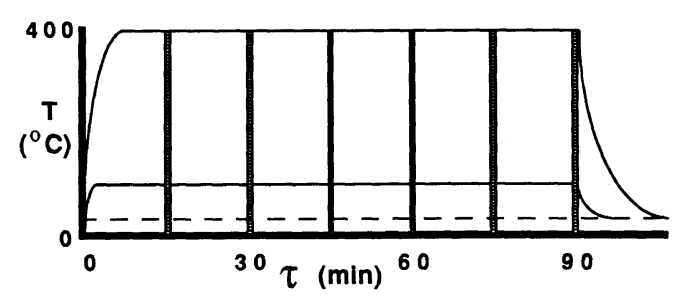

(b)

Figure 3. Procedure used to obtain micrographs as functions of time $(\tau)$ and temperature. Portions of the sample film in a typical grid square (a) are photographed at intervals of $15 \mathrm{~min}$. During the interval, the electron beam (filament) is turned off. The temperatures of interest are shown as a function of $\tau$ in (b), with the beam-on periods shown as vertical bars. Experiments are run for a total of $90 \mathrm{~min}$ at each of four temperatures: $100^{\circ} \mathrm{C}, 212^{\circ} \mathrm{C}, 324^{\circ} \mathrm{C}$, and $400^{\circ} \mathrm{C}$. 
sistant to radiation and high-temperature degradation. ${ }^{25,32}$ Staining, too, was unnecessary due to sufficient contrast between the microphases.

\section{Electron Microscopy}

Bright-field electron micrographs of the ultrathin films in static mode (SBS and MT3) and in dynamic mode, undergoing annealing (SBS) and curing (MT3), were obtained using a JEOL JEM 100CX electron microscope (JEOL Ltd., Peabody, MA 01960) at an accelerating voltage of $80 \mathrm{keV}$. The micrographs revealed microstructure magnified by $26000 \times$ and $33000 \times$ for the SBS copolymers and $50000 \times$ for the MT3 copolymer. Evaporating NMP solvent molecules from MT3 were trapped within the microscope's decontamination chamber, cooled with liquid nitrogen.

The strategy for observing the in-situ heating of the copolymer ultrathin films from ambient temperature to $400^{\circ} \mathrm{C}$ using a JEOL heating-stage attachment is depicted in Figure 3 . When samples were loaded into the microscope, reference micrographs were taken $(\tau=$ $0 \mathrm{~min}$ in Figure 3a) and the heating stage was set to one of four particular temperatures (as shown in Figure $3 \mathrm{~b}$ with the temperatures being $100^{\circ} \mathrm{C}, 212^{\circ} \mathrm{C}, 324^{\circ} \mathrm{C}$, and $400^{\circ} \mathrm{C}$ ). The reference area was kept in the field of view until thermal drift subsided in 3-7 min, depending on the final temperature. Once the field of view became stable, the filament of the microscope was turned off so as to avoid excess radiation degradation, and the position of view was recorded on the position marker on the microscope. The filament was turned on every fifteen minutes to examine the film, ${ }^{* 1}$ and the position of the field of view within one grid square was moved as a function of time (Figure 3a) for each temperature (Figure 3b). One micrograph was usually obtained at each view, although two were acquired if a "pe- culiar" phenomenon was noticed. The time spent for focusing the field of view and taking the micrograph (i.e., irradiating the film) was approximately $1.5 \mathrm{~min}$, which is also shown in Figure $3 \mathrm{~b}$. Microstructures in films being heated were examined for up to $90 \mathrm{~min}$, and additional micrographs were obtained at times after the samples cooled down to ambient temperature.

\section{RESULTS AND DISCUSSION}

\section{SiIm Multiblock Copolymer}

The spherical domains present in the fully cured MT3 films can be seen in Figure 4. Their darkness (opacity to electron beam) suggests they contain the siloxane blocks. Their volume fraction is far less than the $74 \mathrm{wt} \%$ attributed to the soft block, comprised of both the siloxane and diamine segments, so we must conclude that the domains are composed of phase-separated siloxane itself. This would indicate that the remaining portion of the soft

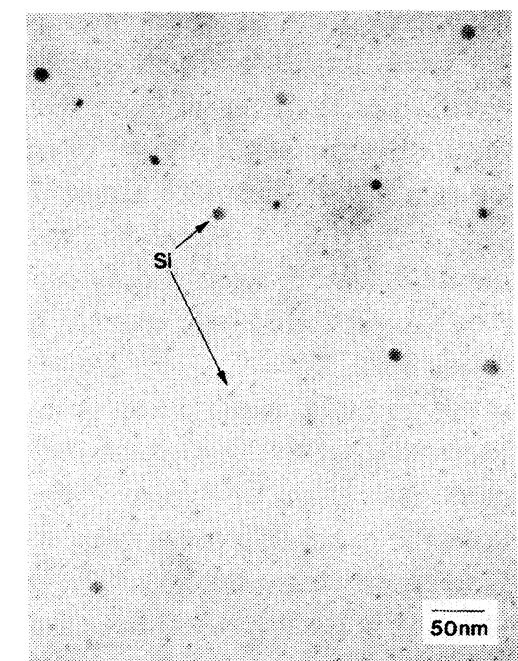

Figure 4. Typical micrograph of the fully cured ${ }^{25}$ MT3 copolymer. Siloxane domains (appearing dark) exhibit a roughly bimodal size distribution with some measuring $2-6 \mathrm{~nm}$ and others about $16 \mathrm{~nm}$ in diameter.

*1 Care was taken to avoid (a) damaging components within the microscope (e.g. the filament and the O-rings) and (b) degrading the films under the electron beam. ${ }^{33}$ 
block - the diamine and the $R_{2}$ groupsurrounds the observed domains but is not visually distinguishable from the imide hard block in TEM. This identification of the dark domains as being siloxane is supported by the observed in-situ response of these domains to tensile strain, in which they deformed significantly over the course of the experiment. ${ }^{23}$

As is evident from Figure 4, the siloxane domains appear to have a roughly bimodal size distribution, with small domains measuring approximately $2-6 \mathrm{~nm}$ in diameter and the larger ones measuring about $16 \mathrm{~nm}$. Two properties of this material may be responsible for this size distribution. First, the copolymer polydispersity index $\left(\bar{M}_{w} / \bar{M}_{n}\right)$ is about 2.0. For a normally broad molecular size distribution with a polydispersity of 2.0 , one might well expect not to observe monodisperse domain sizes. However, one would not expect a bimodal distribution either; a broad domain size distribution might be plausible. The latter expectation is clearly wrong in the present case, and moreover proved to be wrong in another case: ${ }^{34}$ a polydisperse SI copolymer was observed to have almost uniform domain sizes (somewhat larger than the domain size found with a monodisperse SI copolymer of the same $\left.\bar{M}_{n}\right)$. One might still speculate about the consequences of having a blend of two SiIm copolymers whose $\bar{M}_{n}$ were widely separated, though the sample tested here was not believed to be of this sort.

The other potential explanation is suggested by the molecular structure of these multiblock copolymers. As shown in Figure 5, the multiblock molecule is capable of forming spherical domains in more than one way. The smallest such domain (Figure 5a) would have a uniform composition and be of a size scale comparable to that of a triblock; it would also be easiest to form and therefore the most numerous type. The second most numerous type would be the "encapsulated" one (Figure 5b), clearly much bigger. Other forms, with various degrees of lamination, would be so rare than they need

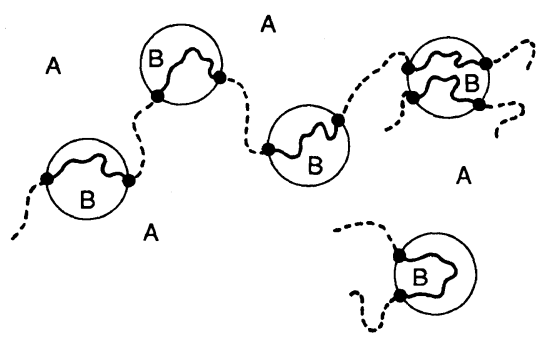

(a)

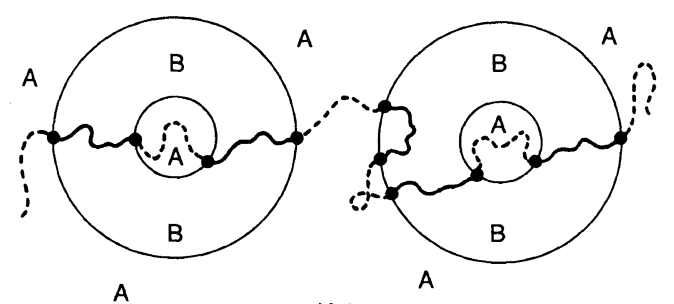

(b)

Figure 5. Schematic representation of possible block configurations in a multiblock copolymer. The domains in (a) possess a uniform composition and, although more intricately connected, resemble those of a triblock. In the case of the "encapsulated" domains displayed in (b), the domains are much larger than those in (a).

not be considered. In all cases, the domains are connected with each other and would produce a solid-like material. The interconnected nature of the microstructures in this multiblock copolymer, discussed at length elsewhere, ${ }^{23}$ is evident in its bulk properties-e.g., the stressstrain properties of this copolymer after the peak stress demonstrate extended flow in fullycured samples (complete microstructural development) and failure in partially-cured samples (no microstructural development) ${ }^{23}$ and will become more evident below.

A partially cured MT3 film is shown in Figure 6 at room temperature and $\tau=0 \mathrm{~min}$. Discrete domains are not present. Instead, we see mottled regions which do not form any regular pattern. There are two possible explanations for certain large regions appearing darker: (1) the regions are thicker than the surrounding areas, or (2) the regions are composed of elements with higher atomic numbers 


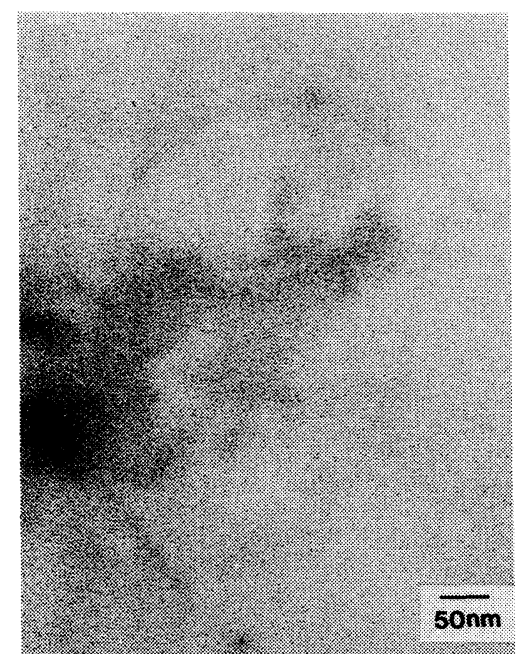

Figure 6. TEM micrograph of the partially cured MT3 copolymer (see the text for cure times) at $\tau=0 \mathrm{~min}$. No discrete siloxane microstructures are evident; instead, mottled features indicate regions of either (a) increased thickness or (b) elements "heavier" than carbon (e.g., Si).

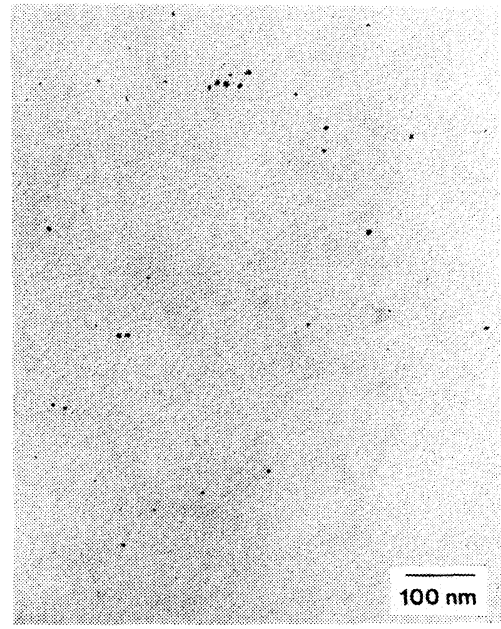

Figure 7. MT3 begins to develop microstructures when cured for $90 \mathrm{~min}$ at $100^{\circ} \mathrm{C}$. The domains are not as numerous and evenly spaced as those in the fully-cured sample (Figure 4).
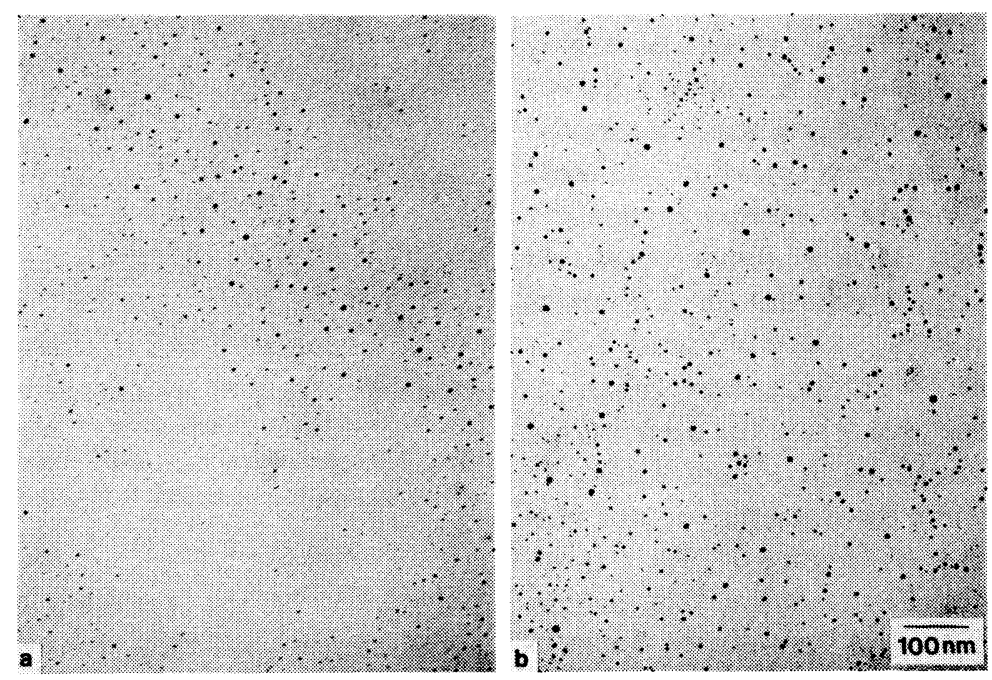

Figure 8. Heated to $212^{\circ} \mathrm{C}$, which corresponds closely to the upper temperature of the manufacturer's suggested cure cycle, ${ }^{25}$ MT3 shows signs of increased phase separation. At $\tau=15 \mathrm{~min}$ (a), domains are prevalent in some areas, but other regions remain homogeneous. In (b), the domains are found everywhere at $\tau=90 \mathrm{~min}$ and resemble those in the fully-cured films (Figure 4); however, the domains do not seem to possess a bimodal size distribution when cured in the microscope.

(e.g., $\mathrm{Si}$ ) than the surrounding regions. All of the reference micrographs of uncured polymer were free of domains, meaning that mi- crophase separation had not occurred; presumably this was due to the presence of a nontrivial amount of NMP. ${ }^{31}$ 


\section{R. J. Spontak and M. C. Williams}

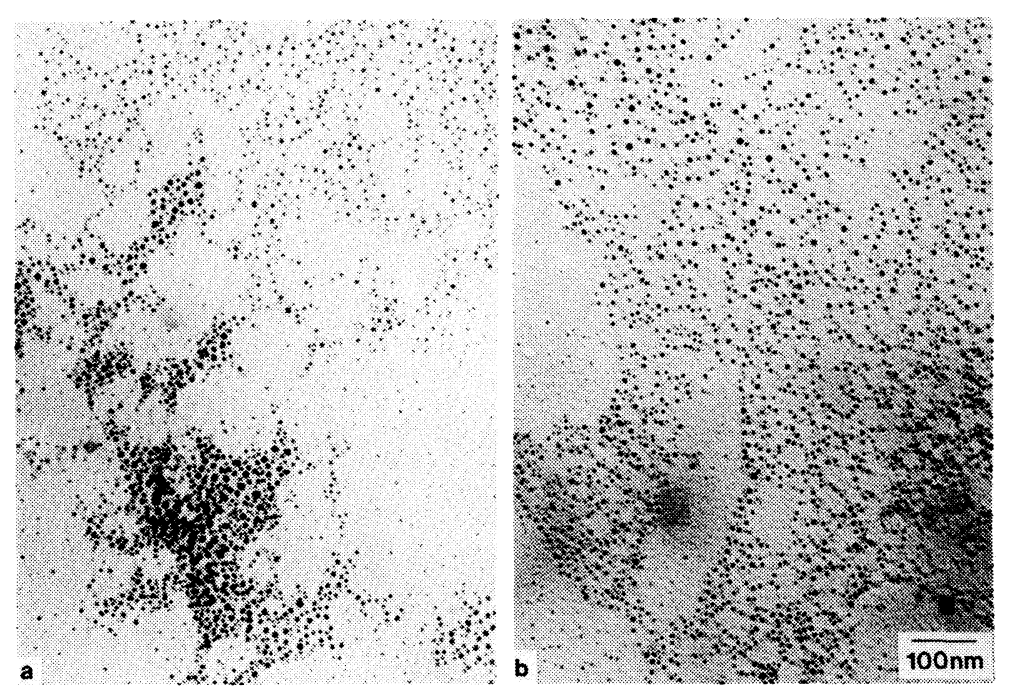

Figure 9. Domain coalescence is observed in these micrographs of MT3 at $324^{\circ} \mathrm{C}$. Large grain boundaries are noticed at $15 \mathrm{~min}(\mathrm{a})$ and $30 \mathrm{~min}(\mathrm{~b})$.

When the MT3 film is cured for $90 \mathrm{~min}$ at $100^{\circ} \mathrm{C}$ (which is above the observed $T_{\mathrm{g}}$ of $61^{\circ} \mathrm{C}^{23}$ ) some siloxane microstructures do appear (Figure 7), but they are not as evenly distributed as in Figure 4. Scanning adjacent fields of view at this time and temperature did not reveal domain concentration different from Figure 7. Domain formation at shorter $\tau$ was not detected at this temperature. At $212^{\circ} \mathrm{C}$, however, the concentration of domains increases dramatically, as seen in Figure 8. For example, Figure 8a, which was taken at $15 \mathrm{~min}$, shows one area where the domains are almost as developed as in Figure 4 and an adjacent area where the domains are not very well developed at all (Figure 7). As $\tau$ is increased to $90 \mathrm{~min}$ (Figure $8 \mathrm{~b}$ ) at this temperature, the entire field of view shows domains resembling those in the fully-cured film (Figure 4), with a continuous, rather than bimodal, size distribution present. It is of interest to note that this time and temperature correspond closely to the end of the manufacturer's suggested cure cycle ${ }^{25}\left(1 \mathrm{~h}\right.$ at $\left.200^{\circ} \mathrm{C}\right)$.

In Figure 9, the temperature is now increased to $324^{\circ} \mathrm{C}$ (which is well within the decomposition-temperature range of this co-

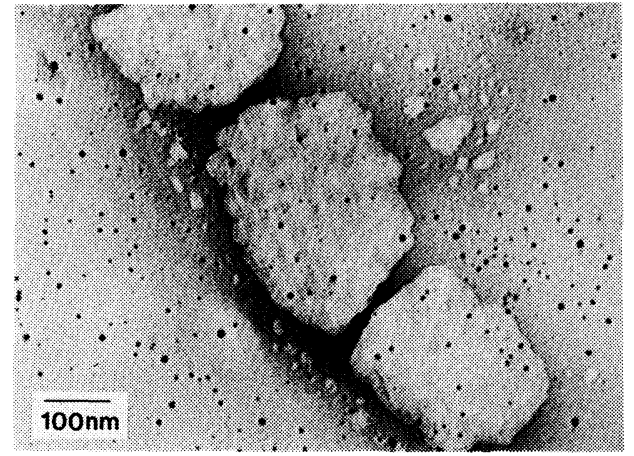

Figure 10. Degraded areas caused by radiation from the electron beam are seen as large "blotches" in the MT3 film. This phenomenon is much different than the domain aggregation observed in Figure 8.

polymer ${ }^{23,25}$, the resulting domains once again appear, but they seem even more concentrated in some areas than in Figures 4 and 8. For example, at $\tau=15 \mathrm{~min}$ (Figure 9a), the domains have a broad continuous size distribution and appear to have coalesced. This is also the case when $\tau$ is increased to $30 \mathrm{~min}$ (shown in Figure 9b).

Several possibilities exist for explaining the increased number and proximity of domains seen in Figure 9. The first is that the film is 
decreasing in size (thickness and length/width) due to NMP evaporation. According to Davis et al. ${ }^{31}$ some Silm films decrease $25-30 \%$ upon complete removal of NMP. Since the supplier reports ${ }^{25}$ that NMP is fully removed during the suggested cure cycle, film shrinkage would be expected in Figure 4 (and 8) and would account for the increased domain concentration relative to Figure 7 . However, no additional shrinkage should occur at a higher temperature (i.e., $324^{\circ} \mathrm{C}$ ), which eliminates film contraction as an explanation for the behavior seen in Figure 9.

The second possibility is that the film is degrading due to exposure to the electron beam at elevated temperatures. An example of a radiation-degraded region (obtained at $324^{\circ} \mathrm{C}$ for $75 \mathrm{~min}$ ) is presented in Figure 10. "Regular" siloxane domains are visible both inside and outside the degraded areas, which are seen as light patches varying in size and shape. Clearly, though, degradation induced by radiation results in patterns much different from the domain agglomerations seen in Figure 9. A third possibility is that the film is thermally decomposing, since $324^{\circ} \mathrm{C}$ is in the temperature regime where decomposition was found with DSC. ${ }^{23}$ If decomposition is occurring, then it appears that the siloxane domains are decomposing since the imide block retains its mechanical properties at elevated temperatures. ${ }^{23,24}$ The microstructural network established by the siloxane domains will be affected by this transition, in which case the crystalline imide matrix may be placed under enough stress so that it loses some degree of its crystallinity, perhaps by partially dissolving in the diamine portion of the soft segment, thereby reducing any hard-soft ordered structure. This would facilitate the coalescence of siloxane domains in a softened "matrix."

This last explanation was substantiated by obtaining diffraction patterns (Figure 11) of the imide at various stages of crystalline order. The pattern of the imide in a fully-cured film is shown in Figure 11a. Upon heating a partiallycured film for $60 \mathrm{~min}$ at $324^{\circ} \mathrm{C}$, we obtained a pattern (Figure 11b) with fewer diffraction spots, indicating a change in lattice structure. After the same film was heated for a total of $90 \mathrm{~min}$ and then allowed to cool to ambient temperature for $12 \mathrm{~h}$, the pattern given in
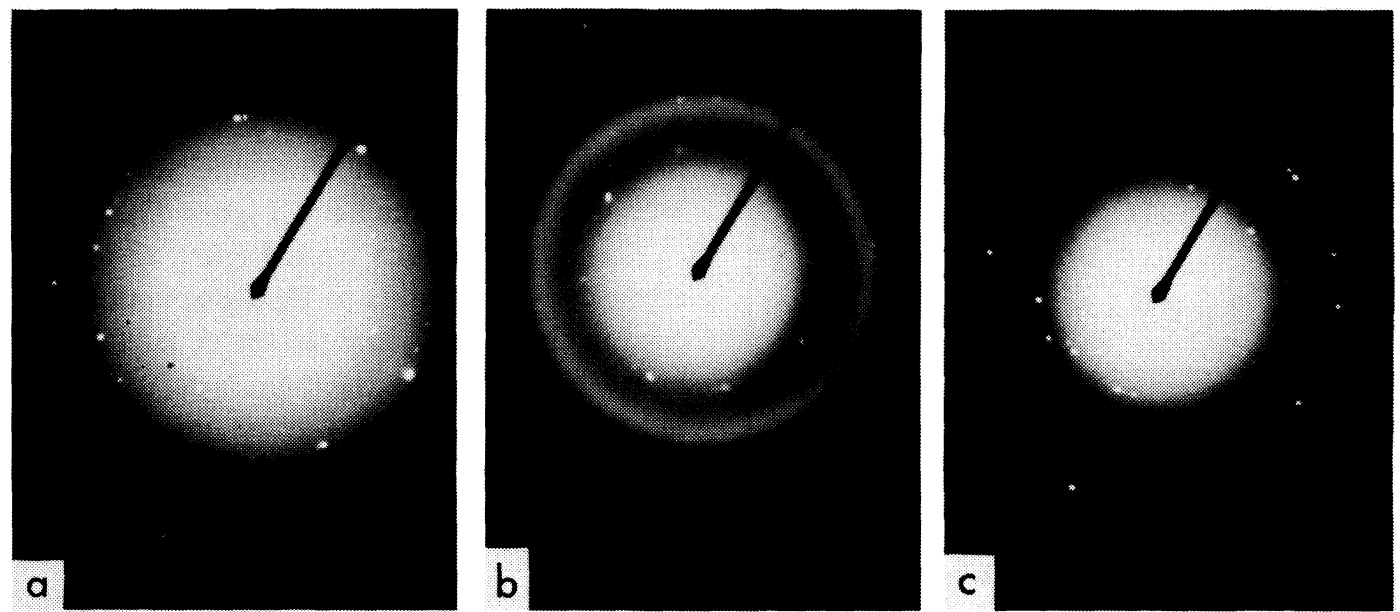

Figure 11. Diffraction patterns (all obtained at a camera length of $120 \mathrm{~cm}$ ) showing the crystalline nature of the imide block in the MT3 multiblock copolymer. The pattern in (a) is taken from a fully-cured ${ }^{25}$ film. The pattern in (b) is obtained from a partially-cured film cured at $324^{\circ} \mathrm{C}$ for $60 \mathrm{~min}$. The decreased clarity and number of diffraction spots is indicative of a structural change in the imide matrix. When the MT3 sample was heated further $(\tau=90 \mathrm{~min})$ and then allowed to cool to room temperature (for $12 \mathrm{~h})$, the imide seems to regain a substantial degree of its crystallinity (c). 
Figure 11c resulted. Upon comparing Figures $11 \mathrm{a}$ and $11 \mathrm{c}$, we see that the diffraction spots in Figure $11 \mathrm{c}$ resemble those in Figure 11a rather closely, both in sharpness and number, suggesting that the imide regained its crystalline structure when it was allowed to return to a temperature below the decomposition temperature.

\section{Styrene-Butadiene Triblock Copolymers}

I. Theory. In the last section, we presented evidence for the in-situ curing of the MTs multiblock copolymer. Here, we turn our attention to both the degree of non-equilibrium in the SBS copolymer films and their subsequent annealing. All of these copolymers microphase separate at room temperature if allowed to equilibrate. ${ }^{1-3}$ In addition, the temperature of phase separation in these copolymers is also predicted from the theory of Williams and co-workers ${ }^{3,14,15}$ (Figure 12). From the minimum in free energy upon microphase separation $\left(\Delta G_{\min }\right)$ and the parameters which correspond to $\Delta G_{\min }=0$, we can determine $T_{\mathrm{s}}$ by

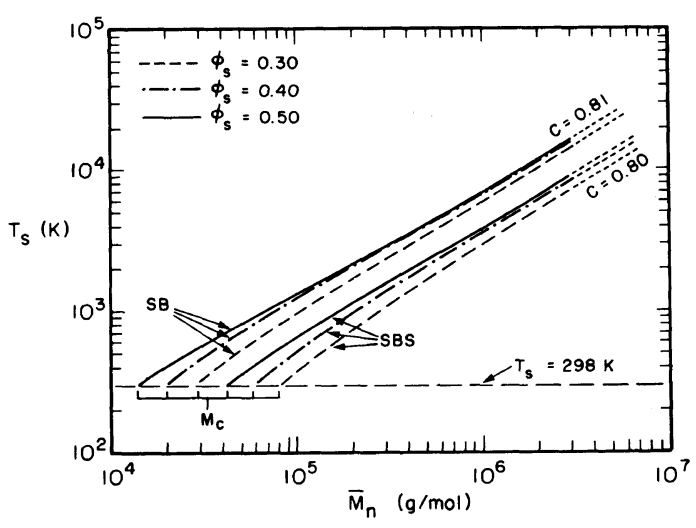

Figure 12. The microphase-separation temperature $\left(T_{\mathrm{s}}\right)$ predicted as a function of molecular weight $\left(\bar{M}_{n}\right)$. For both SB and SBS copolymers, $T_{\mathrm{s}}$ is predicted to scale with $\bar{M}_{n}{ }^{c}$, where $c=0.81$ for diblocks and 0.80 for triblocks. In addition, increasing the bulk PS volume fraction $\left(\phi_{\mathrm{S}}\right)$ from 0.30 (dashed line) to 0.40 (dot-dashed line) to 0.50 (solid line) results in a higher $T_{\mathrm{s}}$ at constant $\bar{M}_{n}$, and a lower critical molecular weight of phase separation, $M_{\mathrm{c}}$, at constant $T_{\mathrm{s}}\left(T_{\mathrm{s}}=298 \mathrm{~K}\right.$, here $)$.

$$
T_{\mathrm{s}}=\frac{\Delta H}{\Delta S}
$$

where $\Delta H$ and $\Delta S$ account for differences in the energetic and configurational contributions, respectively, between the structured (phaseseparated) and homogeneous states in the copolymer system. The explicit forms of both $\Delta H$ and $\Delta S$ are provided in detail elsewhere. ${ }^{3,14,15}$

In Figure 12, $T_{\mathrm{s}}$ is seen to increase as a function of molecular weight over the range from the critical molecular weight for phase separation $\left(M_{\mathrm{c}}\right)$ to $\bar{M}_{n} \approx 3000000 \mathrm{~g} \mathrm{~mol}^{-1}$ for both the diblock and triblock architectures; this predicted behavior is in qualitative agreement with the experimental findings of Kraus and Hashimoto. ${ }^{4}$ The almost-linear relationship between $\log T_{\mathrm{s}}$ and $\log \bar{M}_{n}$, especially at high $\bar{M}_{n}\left(>M_{c}\right)$, is suggestive of an expression of the type

$$
T_{\mathrm{s}}=k \bar{M}_{n}{ }^{c}
$$

where the limiting values of the exponent $c$ for the SB and SBS cases are 0.81 and 0.80 at the high-molecular-weight regimes, respectively. In agreement with data reported on polyetherbased polyurethanes, ${ }^{12}$ Figure 12 also indicates that $T_{\mathrm{s}}$ increases (up to a point) with an increase in the bulk volume fraction of the hard styrene component $\left(\phi_{\mathrm{S}}\right)$. This increase is predicted to be more dramatic for the SB architecture than the SBS (Figure 12).

The $T_{\mathrm{s}}$ predictions from Figure 12 are not completely applicable to the three copolymers used in this study, since the latter are far from being monodisperse (the highest polydispersity index is 2.0, for the F411 grade, given in Table I). Theories ${ }^{35-37}$ considering the effects of polydispersity on block copolymer properties have been recently proposed; one ${ }^{36,37}$ predicts that $T_{\mathrm{s}}$ at constant $\bar{M}_{n}$ increases as a function of polydispersity. This means that if phase separation is favorable for a monodisperse polymer, then it is even more favorable for a polydisperse copolymer (having the same $\bar{M}_{n}$ ) to phase separate under the same conditions of 


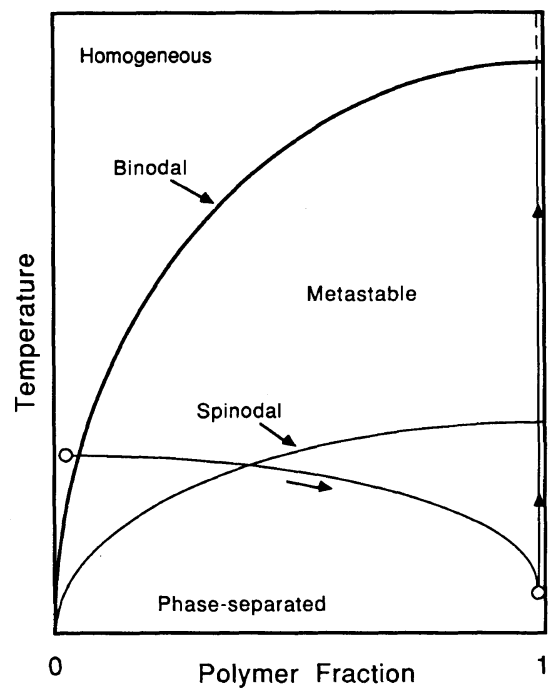

Figure 13. Schematic of the phase diagram for block copolymers in solution. The binodal line corresponds to the onset of phase separation $\left(T_{\mathrm{s}}^{29}\right)$, and the spinodal line refers to phase instability. Homogeneous samples are prepared in dilute solutions at an elevated temperature so as to guarantee phase homogeneity (circle at left). Upon rapid solvent removal and thermal equilibration (lower arrowed curve), the resultant polymer films are in a state of non-equilibrium (circle in lower right). These films are then heated (right arrowed line) up to temperatures as high as $400^{\circ} \mathrm{C}$, which may extend into the metastable or homogeneous regions.

bulk composition, chemical difference, and molecular architecture.

Predictions for $T_{\mathrm{s}}$ from Figure 12 indicate that microphase separation is the favored equilibrium state at room temperature for the copolymers used in this study. However, the experimental objective here is to "freeze" the microstructures in a non-equilibrium state and then observe their response to elevated temperatures. This process is depicted schematically in Figure 13, where the polymer fraction refers to the volume fraction of copolymer in solution. The binodal $\left(T_{\mathrm{s}}\right)$ and spinodal curves represent phase separation behavior both observed ${ }^{7,27}$ and predicted. ${ }^{28}$ Phase homogeneity in the copolymer (lower left circle) is guaranteed by having a dilute solution $(\approx 0.5 \%)$ at an elevated temperature $\left(90^{\circ} \mathrm{C}\right)$. When the cast ultrathin films are subjected to vacuum, the toluene solvent is rapidly removed (lower arrowed curve) until an almost solvent-free copolymer film remains (lower right circle). The degree of non-equilibrium $\left(\Delta_{\mathrm{ne}}\right)$ in each resulting film depends primarily on the fraction of PS present, since the PS block glassifies at room temperature and thus "locks in" whatever state prevails. The concept of $\Delta_{n e}$, which is completely qualitative, is useful in categorizing the copolymers according to material properties-e.g., composition, molecular weight, and polydispersity ${ }^{2}$ - which will increase the extent of non-equilibrium in the ultrathin films. For instance, from knowing $\phi_{\mathrm{S}}$ and preparing all of the sample films in the same manner, we expect a priori that $\Delta_{\mathrm{ne}}(\mathrm{F} 414)>\Delta_{\mathrm{ne}}(\mathrm{F} 411) \approx \Delta_{\mathrm{ne}}(\mathrm{F} 416)$, where the $\mathrm{PS}$ fractions of each copolymer grade are listed in Table I.

In addition to being polydisperse, the copolymers to be studied have been stained with osmium tetroxide $\left(\mathrm{OsO}_{4}\right)$ to enhance contrast between the microphases in TEM. The stain, which also acts as a stiffening agent, will significantly alter the kinetics of (a) phase separation in those copolymers with large $\Delta_{n e}$-where little, if any, initial phase separation occurred - and (b) domain evolution in those which had smaller $\Delta_{\mathrm{ne}}$-where some initial phase separation did occur. The kinetics of phase separation scale as the viscosity, ${ }^{38}$ or the relaxation time of the polymer molecules; and the viscosity of a polymer is directly related (by the Doolittle equation ${ }^{39}$ ) to the free volume present. Since the staining molecules occupy a fraction of this previously unoccupied volume, ${ }^{3}$ we can calculate, at least semiquantitatively, the free-volume fraction remaining after staining (and the corresponding viscosity). Knowing the relationship between the free-volume fraction present before and after staining, and the corresponding viscosities, will facilitate determining the degree to which the $\mathrm{OsO}_{4}$ slows the phase-separation and domain-evolution processes.

Since the PB constitutes the rubbery matrix, 
most, if not all, of the free volume governing diffusion during phase separation will be found in the PB matrix. The free volume fraction $\left(\Phi_{\mathrm{f}}\right)$ in PB can be determined as a function of temperature by the expression: ${ }^{39}$

$$
\Phi_{\mathrm{f}}=\Phi_{\mathrm{g}}+\alpha_{\mathrm{T}}\left(T-T_{\mathrm{g}}\right)
$$

where $\Phi_{\mathrm{g}}$ is the free-volume fraction in a glassy polymer $(\approx 0.025)$ and $T_{\mathrm{g}}$ is the glasstransition temperature (taken as $193.15 \mathrm{~K}$ for polybutadiene). The thermal expansion coefficient for free volume, $\alpha_{\mathrm{T}}$, is $7 \times 10^{-4} /{ }^{\circ} \mathrm{C}$, as determined by data on 1,4-polybutadiene. ${ }^{40}$ Assuming that the fraction of free volume remaining after staining, $\Phi_{\mathrm{f}}{ }^{\prime}$, varies linearly with the fraction of free volume occupied by the staining molecules, $\Phi^{\prime}$, we obtain

$$
\Phi_{\mathrm{f}}{ }^{\prime}\left(\Phi^{\prime}, T\right)=a(T)-b(T) \Phi^{\prime} \Phi_{\mathrm{f}}(T)
$$

where $a$ and $b$ are temperature-dependent parameters and $\Phi_{\mathrm{f}}$ is the free-volume fraction in the absence of any stain.

Modification of the Doolittle equation ${ }^{39}$ yields

$$
\eta^{\prime}=A(T) \exp \left(B(T) / \Phi_{\mathrm{f}}{ }^{\prime}\right)
$$

where $\eta^{\prime}$ is the viscosity of the stiffened rubber and $A(T)$ and $B(T)$ are temperature-dependent parameters. Derivation of an equation similar to the WLF equation ${ }^{39}$ (provided in the appendix) results in

$$
\ln \eta_{\mathrm{r}}=B(T)\left[\frac{1}{\Phi_{\mathrm{f}}{ }^{\prime}}-\frac{1}{\Phi_{\mathrm{f}}}\right]
$$

where $\eta_{\mathrm{r}}$ is defined by

$$
\eta_{\mathrm{r}} \equiv \frac{\eta^{\prime}\left(T, \Phi^{\prime}\right)}{\eta(T)}
$$

The ', once again, refers to stain conditions. Values of $\Phi_{\mathrm{f}}(T)$ and $B(T)$ are presented as functions of temperature in Table II.

The relationship between $\eta_{\mathrm{r}}$ and $\Phi_{\mathrm{f}}{ }^{\prime}$ is presented in Figure 14, with $\eta_{\mathrm{r}}=1$ corresponding to conditions in the absence of stain. This indicates that the viscosity ratio (or relaxationtime ratio), taken with respect to no-stain
Table II. Parameters used to predict the effects of $\mathrm{OsO}_{4}$ stain on phase-separation kinetics

\begin{tabular}{ccc}
\hline $\mathrm{Temp} /{ }^{\circ} \mathrm{C}$ & $\Phi_{\mathrm{f}}^{\mathrm{a}}$ & $B(T)^{\mathrm{b}}$ \\
\hline 25 & 0.0985 & 0.9022 \\
100 & 0.1510 & 0.9352 \\
212 & 0.2294 & 0.9577 \\
324 & 0.3078 & 0.9691 \\
400 & 0.3610 & 0.9741 \\
\hline
\end{tabular}

a Calculated from eq 3.

b Calculated from eq A7 and eq A9 (in the appendix).

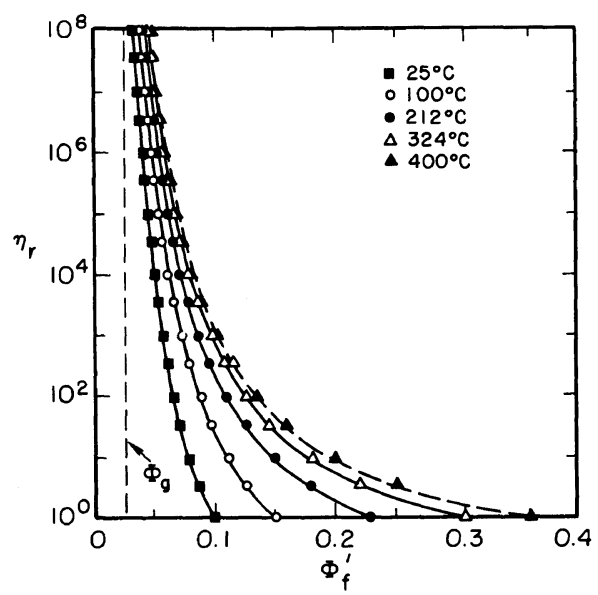

Figure 14. The ratio of viscosities, or molecular relaxation times, in stained and unstained PB $\left(\eta_{\mathrm{r}}\right)$ as a function of the free-volume fraction remaining after stain is applied $\left(\Phi_{\mathrm{f}}\right)$ at five temperatures: $25^{\circ} \mathrm{C}(\square)$, $100^{\circ} \mathrm{C}(\bigcirc), 212^{\circ} \mathrm{C}(\bigcirc), 324^{\circ} \mathrm{C}(\triangle)$, and $400^{\circ} \mathrm{C}(\Delta)$. The dashed line at $400^{\circ} \mathrm{C}$ indicates that thermal decomposition may be occurring in the sample. As $\Phi^{\prime} \rightarrow 1.0$, the rubber is assumed to behave like a glass, with $\Phi_{\mathrm{f}}^{\prime} \rightarrow \Phi_{\mathrm{g}}$.

conditions, is very sensitive to the free volume available for diffusion. For example, a slight decrease in $\Phi_{\mathrm{f}}{ }^{\prime}$ from 0.11 to 0.07 at $100^{\circ} \mathrm{C}$ increases $\eta_{\mathrm{r}}$ by two orders of magnitude. The model proposed here is not meant to yield accurate quantification of the $\mathrm{OsO}_{4}$ staining of PB. Instead, it is designed to obtain the order of magnitude at which diffusion-limited phase separation will occur in these stained block copolymers. Baumgartner and Heermann, ${ }^{41}$ using Monte Carlo simulations, have shown that phase separation occurs in polymer melts in times on the order of $0.01 \mathrm{~s}$. Since phase 
separation and domain development occur during about $20 \mathrm{~min}$ (or $1200 \mathrm{~s}$ ) in the stained copolymers here, then $\eta_{\mathrm{r}} \approx 10^{5}$, indicating that $0.05<\Phi_{\mathrm{f}}{ }^{\prime}<0.10$.

The values of $T_{\mathrm{s}}$ are not expected to be the same in these stained films as in their bulk counterparts for two additional reasons. The first is that the staining not only hinders diffusional mobility during phase separation but also increases the chemical dissimilarity between the blocks. According to the LearyHenderson-Williams theory, ${ }^{14,15} T_{\mathrm{s}} \propto(\Delta \delta)^{2}$, in which $\Delta \delta$ is the difference in solubility parameters of the two blocks. As the $\mathrm{OsO}_{4}$ chemically bonds to the $\mathrm{PB}$, it changes the solubility of that block, probably by increasing the solubility parameter of the osmium-modified PB. Since the blocks are then even more thermodynamically incompatible, $T_{\mathrm{s}}$ increases. The second reason is that phase-separation temperatures are indeed affected by the thinness of polymeric films ${ }^{42,43}$; this is most likely due to an increase in surface-tension effects at the air/polymer interface (discussed later).

II. Annealing. The first SBS copolymers to be presented are the samples exhibiting cylindrical morphology (F411 and F416), each copolymer composed of $30 \mathrm{wt} \%$ PS. The microstructural formation induced in the F411 grade, which has the highest molecular weight (115000 $\mathrm{g} \mathrm{mol}^{-1}$ ) and polydispersity index (2.00) of all the samples studied here, is shown in Figure 15. Phase separation is apparent from the mottled structure present, wherein

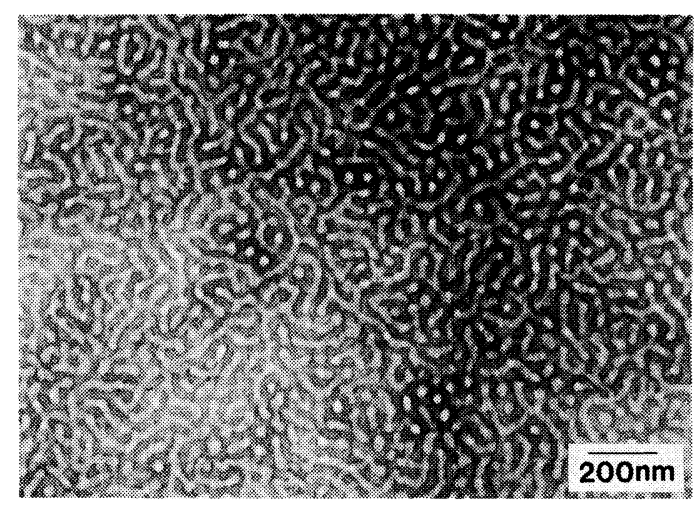

Figure 15. Micrograph of the phase separation in the F411 SBS (30wt \% PS) block copolymer upon initial casting. Diffuse domain boundaries and lack of ideal cylindrical morphology (observed in equilibrated samples $^{2,3,26}$ ) are evidence that this film is in a state of nonequilibrium.
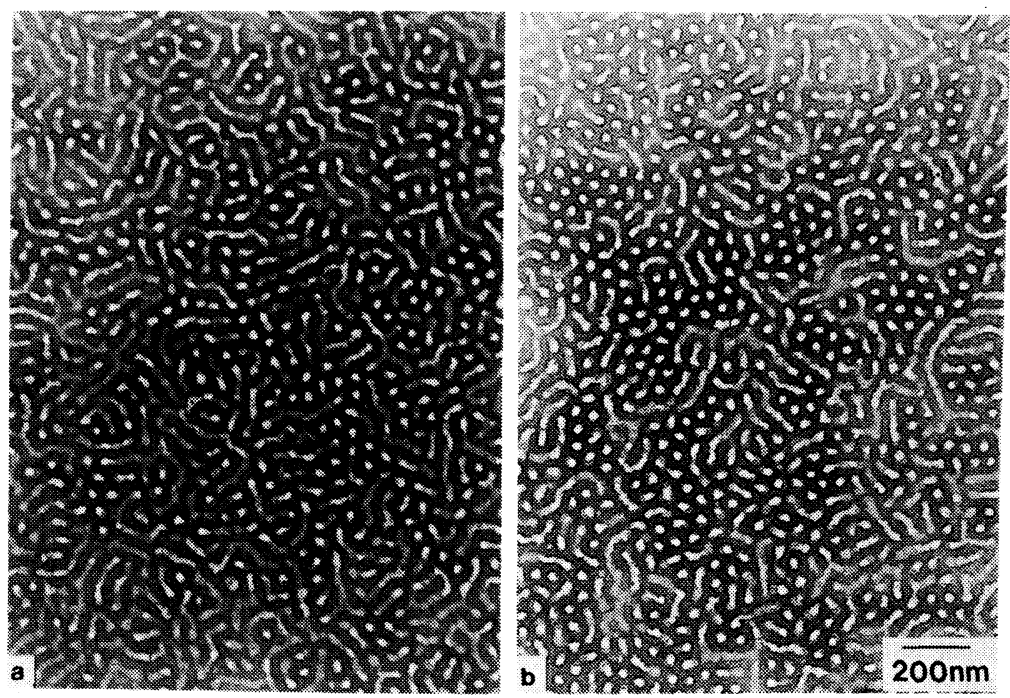

Figure 16. Upon heating the $\mathrm{F} 411$ copolymer at $212^{\circ} \mathrm{C}$, for $15 \mathrm{~min}$ (a) and $30 \mathrm{~min}$ (b), two-dimensionally circular (end-on cylindrical) domains become more prominent than in the as-cast film (Figure 15). In fact, some hexagonal packing is observed in (b). 


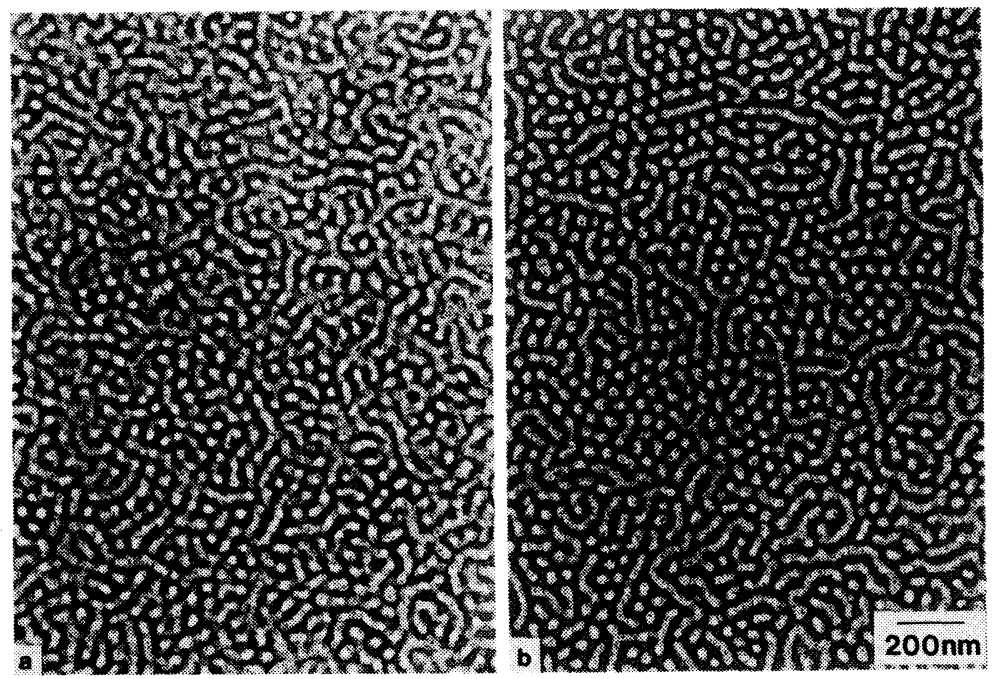

Figure 17. Effects of annealing F411 for $\tau=15 \mathrm{~min}$ (a) and $45 \mathrm{~min}$ (b) at $324^{\circ} \mathrm{C}$. End-on cylindrical PS domains have developed with sharper domain boundaries than those in Figure 15.

the light areas correspond to the dispersed PS phase and the dark areas correspond to the stained PB matrix. Dispersed domains are not regular-i.e., hexagonally packed-and do not clearly represent cylinders (although the presence of two-dimensional circles is suggestive of cylinders oriented normal to the film). In addition, the domain boundaries appear somewhat diffuse, similar to domain boundaries in non-equilibrated styrene-isoprene branched block copolymers. ${ }^{4}$ Diffuse boundaries are evidence that the phase separation is a gradual phase-demixing, the reverse of which - gradual intermixing upon phase homogenization-has been reported. ${ }^{5}$

Upon heating the non-equilibrated F411 to $212^{\circ} \mathrm{C}$ (Figure 16), we see more of the circular domains and less-diffuse domain boundaries. In Figure 16a, the sample has been heated for $15 \mathrm{~min}$. The number of end-on cylindrical domains, denoted by $N$ and referring to those that appear two-dimensionally circular, has increased from Figure 15. Domain development, after $30 \mathrm{~min}$ of annealing, is responsible for the first signs of hexagonal packing (Figure 16b). Once again, $N$ is seen to increase significantly, thereby indicating that annealing is allowing the copolymer to attain its preferred ordering by increasing diffusional mobility (even through the stained PB matrix). Similar trends are observed when as-cast F411 samples are heated to $324^{\circ} \mathrm{C}$ (Figure 17). Diffuse boundaries become more distinct after $15 \mathrm{~min}$ (Figure 17a), and $N$ increases substantially after $45 \mathrm{~min}$ (Figure 17b), with hexagonal ordering now visible.

Quantification of these trends is exceptionally difficult, owing particularly to structural variations that may exist in each ultrathin film. A semi-quantitative approach which uses $N$ seems valid in an effort to characterize the sample in a fashion suited to discussion of general domain evolution. Values of $N$ were obtained for each micrograph by counting the number of domains that appeared both (a) two-dimensionally circular and (b) unconnected to other structures. Since the total micrograph areas $\left(A_{\mathrm{m}}\right)$ averaged $32.54 \mu \mathrm{m}^{2}$ (at a magnification of $26000 \times$ ) and $20.20 \mu \mathrm{m}^{2}$ (33000 $\times$ magnification), values of $N$ representative of the domain structures present have been acquired to yield statistically valid results. ${ }^{* 2}$

\footnotetext{
*2 Values of $N$ for any given micrograph ranged from 16 (at room temperature) to 1036 (at $T=212^{\circ} \mathrm{C}$ ).
} 
The maximum possible number of domains, assuming hexagonal packing, is determined next by knowing the total micrograph area and the area of one unit cell, defined as the hexagonal region encompassing one domain. The area of a unit cell $\left(A_{\mathrm{c}}\right)$ is given by ${ }^{44}$

$$
A_{\mathrm{c}}=(3 \sqrt{3} / 2) s^{2}
$$

where $s$ is the length of one edge of the hexagon. The perpendicular distance from an edge to the midpoint of the hexagon $(r)$ is ${ }^{44}$

$$
r=(\sqrt{3} / 2) s
$$

Upon substituting eq 9 into eq 8 and recalling that $r=D / 2$, where $D$ is the microstructural repeat distance, we obtain

$$
A_{\mathrm{c}}=(\sqrt{3} / 2) D^{2}
$$

For the F411 copolymer, $D \approx 48 \mathrm{~nm}$. With $A_{\mathrm{c}}$ known, the theoretical number of domains that could exist within the entire area of the micrograph $\left(N_{\mathrm{t}}\right)$ can be determined from the ratio $A_{\mathrm{m}} / A_{\mathrm{c}}$. An ordering parameter, $\kappa$, is then defined as

$$
\kappa \equiv \frac{N}{N_{\mathrm{t}}} \times 100 \%
$$

The physical significance of $\kappa$ is that it represents a measure of attainment of equilibrium structure within the films studied here. Figure 18 depicts the relationship between $\kappa$ and time $\tau$ for the F411 copolymer for the four annealing temperatures used in this study. At $100^{\circ}$, little change in $\kappa(\tau)$ indicates that not enough free volume at this temperature is yet available for continued phase separation. This is expected since the temperature is still very close to the glass-transition temperature $\left(T_{\mathrm{g}}^{S}\right)$ of the PS phase $\left(94.2^{\circ} \mathrm{C}^{3}\right)$. Upon cooling from $100^{\circ} \mathrm{C}$, howver, $\kappa$ increases, suggesting that phase separation is somehow enhanced as the temperature decreases from $T_{\mathrm{g}}^{\mathrm{S}}$ to room temperature. At $212^{\circ} \mathrm{C}$ and $324^{\circ} \mathrm{C}$, significant increases in $\kappa(\tau)$ are observed for $\tau \geq 15 \mathrm{~min}$ (Figures 16 and 17). Because the system is still within the thermodynamically incompatible

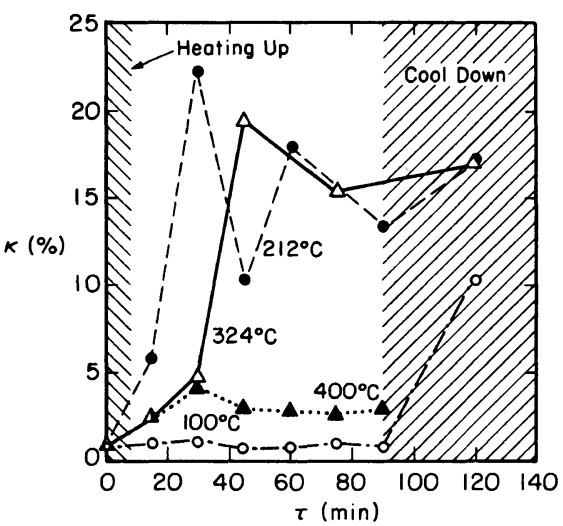

Figure 18. Ordering parameter $\kappa$ as a function of time $(\tau)$ at four temperatures. The value of $\kappa$ reflects the ratio of the number of circular, non-connected PS domains to the theoretical number of hexagonally-packed domains that could form in the area of view. Heating times and cooling times are presented as cross-hatched areas, and descriptions of the observed behavior in $\kappa(\tau)$ are given in the text. No point was obtained at $120 \mathrm{~min}$ for cooling from $400^{\circ} \mathrm{C}$. An evaluation after a full day of cooling from $212^{\circ} \mathrm{C}$ gave $\kappa=31.2 \%(\tau=1,440 \mathrm{~min})$, indicating that structural evolutions can continue over long periods of time, even at room temperature, if triggered by sufficient thermal energy.

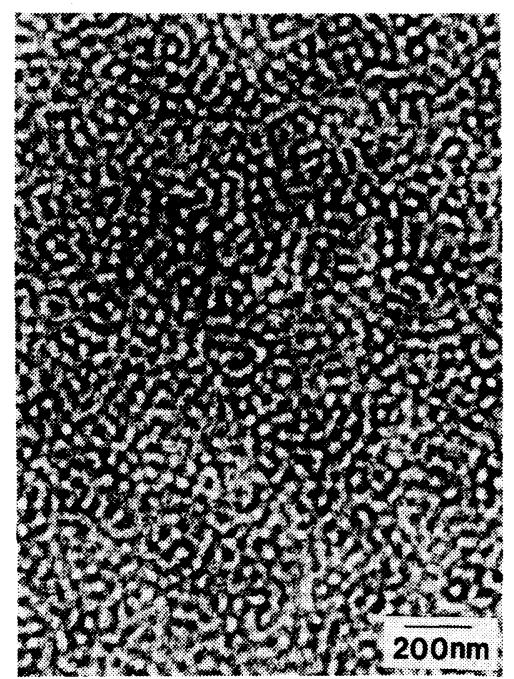

Figure 19. Thermal decomposition in the F411 copolymer at $400^{\circ} \mathrm{C}(\tau=60 \mathrm{~min})$. The distinct domain boundaries are replaced by an overall "charred" appearance. 
region in the styrene-(stained-butadiene) phase diagram, phase separation continues at these elevated temperatures. Once again, $\kappa$ increases as the samples are allowed to cool down; an increase in microstructural ordering upon cooling has also been observed in other studies. ${ }^{4}$

The curve in Figure 18 corresponding to $400^{\circ} \mathrm{C}$ shows no sign of $\kappa$ changing with time. This behavior can be resolved in one of two ways. First, $400^{\circ} \mathrm{C}$ may be beyond $T_{\mathrm{s}}$ (the binodal curve in Figure 13), in which case the copolymer is subjected to no driving force to maintain phase separation but remains as dictated by the rigidified stained matrix. The second explanation is that the film, the PB matrix in particular, is thermally decomposing. Evidence of what is believed to be such decomposition is shown in Figure 19, taken at $60 \mathrm{~min}$. The matrix is much different than in the previous micrographs and appears almost "charred." The mottled pattern observed in samples upon initial film casting (e.g., Figure 15 ) is still present, with little domain development. These two points - the "charred" matrix and the lack of domain evolution-probably

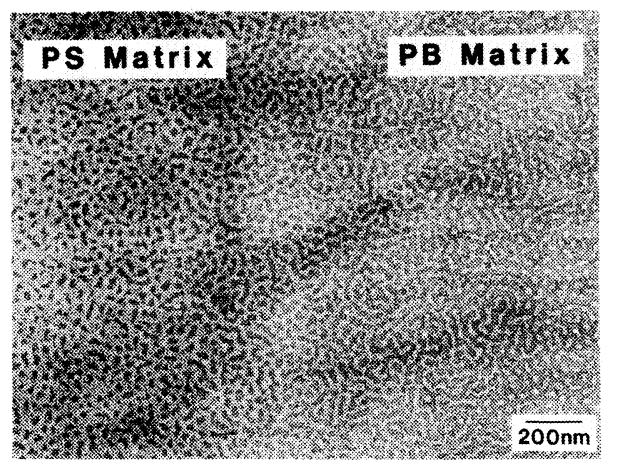

Figure 20. Coexistence of both PB and PS matrices in $\mathrm{F} 411$, annealed to $324^{\circ} \mathrm{C}$ for $75 \mathrm{~min}$. Phase inversion such as this has been reported elsewhere ${ }^{14,45,46}$ and is most likely the result of either local-order kinetic limitations (in which the high-viscosity PS reduces diffusional mobility during phase separation) or local-composition variations (in which the equilibrium free energy may vary enough from point to point that one morphology is thermodynamically-preferred over another adjacent to it). indicate that thermal decomposition of the stained PB matrix is occurring.

One peculiar pattern in the F411 copolymer was observed when the film was heated to $324^{\circ} \mathrm{C}$ for $75 \mathrm{~min}$ (Figure 20). In this case, a light (PS) matrix is seen on the left of the micrograph, and a dark (PB) matrix is seen on the right. Phase inversion such as the example presented here has been reported before. Leary and Williams ${ }^{14}$ showed that phase inversion occurred in a PB-rich SBS copolymer when the copolymer was heated beyond its $T_{\mathrm{s}}$ and then allowed to cool to ambient temperature. Phase inversion has also been reported in etheramide block copolymers ${ }^{45}$ and is discussed elsewhere by Haward. ${ }^{46}$

The thermal response of domains in the other copolymer exhibiting a cylindrical morphology (F416) is somewhat different than in the F411 grade. As is evident from Figure 21, the degree of microstructural formation upon initial film casting is quite high; that is, $\kappa(\tau=$ $0) \approx 65 \%$ (with $D \approx 40 \mathrm{~nm}$ ). The reason for

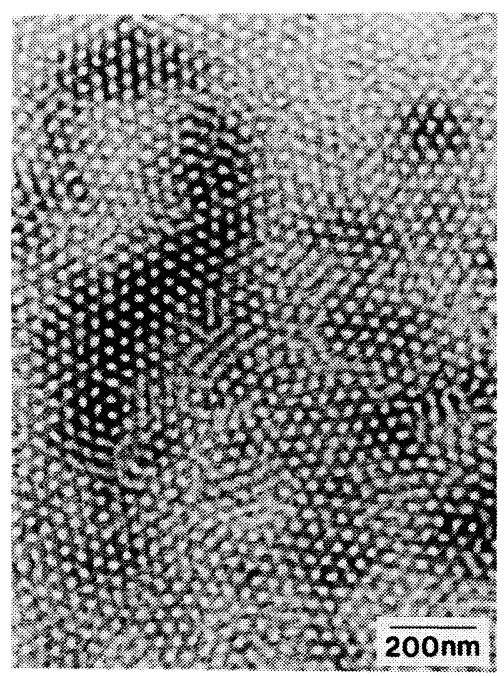

Figure 21. TEM micrograph of the phase-separated morphology in the F416 copolymer (30 wt \% PS) upon initial casting. Such rapid domain development $(\kappa=65 \%$ ) under clearly non-equilibrium conditions can be attributed to its lower molecular weight and polydispersity (and consequent increased diffusional mobility), relative to the $F 411$ grade. 
such a high ordering upon phase separation lies within the physical properties of this copolymer. Upon comparing F416 with F411, we see that, although each is composed of $30 \mathrm{wt} \%$ PS, the molecular weight of F416 is lower (84800 $\mathrm{g} \mathrm{mol}^{-1}$ for F416 and 115000 for F411) and the polydispersity index is lower (1.65 for F416 and 2.00 for F411). Hence, we may conclude that, keeping all aspects of the sample preparation identical, the greater extent of domain ordering in the initial casting of F416 (as compared to F411) is most likely due to increased diffusional mobility favored by lower molecular weight and lower polydispersity. In other words, non-equilibrium effects are more pronounced in systems with high molecular weights and high polydispersity indices, which is in agreement with the findings of Kinning and Thomas. ${ }^{47}$ Further annealing of F416 does not alter the degree of ordering very much, with $\kappa(\tau)$ never differing from $\kappa(0)$ by more than $11 \%\left(\kappa_{\max }=72 \%\right)$.

The copolymer exhibiting lamellar microstructures (F414) behaved much differently when annealed (Figure 22). Initial microstruc- tural formation upon film casting is seen in Figure 22a. Lamellae are observed at $\tau=0 \mathrm{~min}$, but the contrast between phases is weak (as compared to the fully-equilibrated lamellae shown later). Some structure was initially observed, but most of the films appeared homogeneous-i.e., lacking structure (see the left side of Figure 22a). When the film was heated to $100^{\circ} \mathrm{C}$ for $60 \mathrm{~min}$, new lamellar structure was detected (Figure 22b). Original lamellae were distinguished from new structure by the sharpness of the phase separation in the former. Most of the new lamellae seem connected to the original lamellae, thereby enlarging the lamellar region. However, some structure appears to be forming within the initially homogeneous areas.

Similar structural development was observed when F414 films were heated to $212^{\circ} \mathrm{C}$ (Figure 23) and $324^{\circ} \mathrm{C}$ (Figure 24). In Figure $23 \mathrm{a}$, the film was annealed for $15 \mathrm{~min}$ and exhibited signs of lamellar growth. A complex and more ordered lamellar pattern is observed in Figure 23b when the film was further heated for an additional $30 \mathrm{~min}$
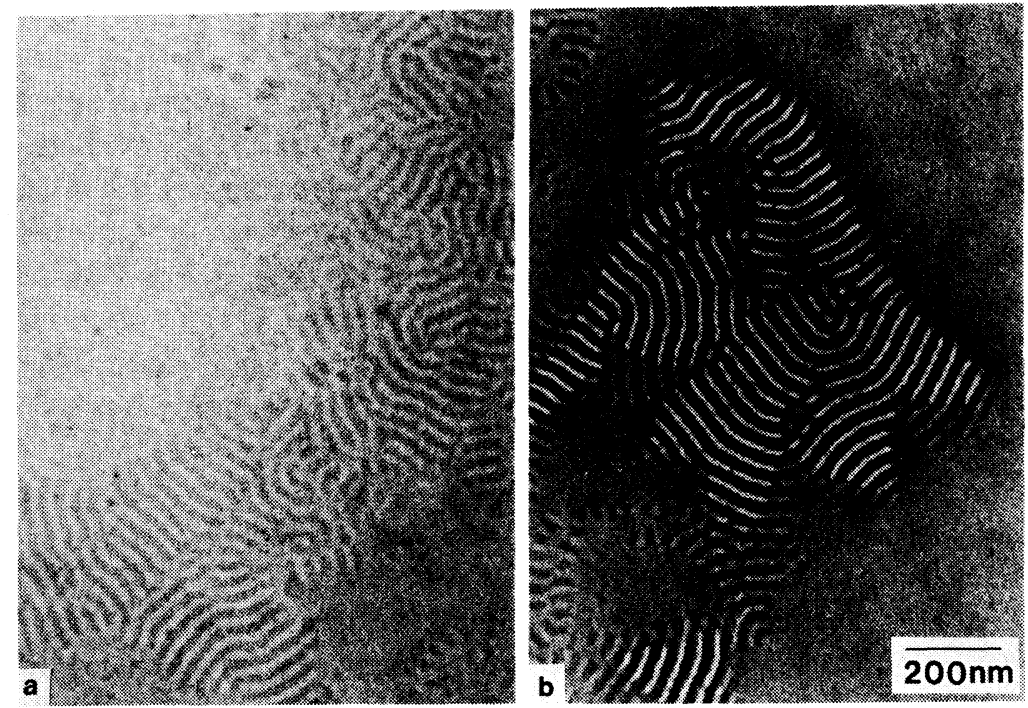

Figure 22. Initial and developing lamellar structures in the F414 copolymer ( $40 \mathrm{wt} \%$ PS) at ambient conditions (a) and at $100^{\circ} \mathrm{C}$ (b). Non-structured regions in (a) indicate phase homogeneity due primarily to the high fraction of PS, which produces mixed regions with $T_{\mathrm{g}}$ so high that diffusional mobility is repressed at room temperature. Some structural development is evident at the elevated temperature, at $\tau=60 \mathrm{~min}$. 

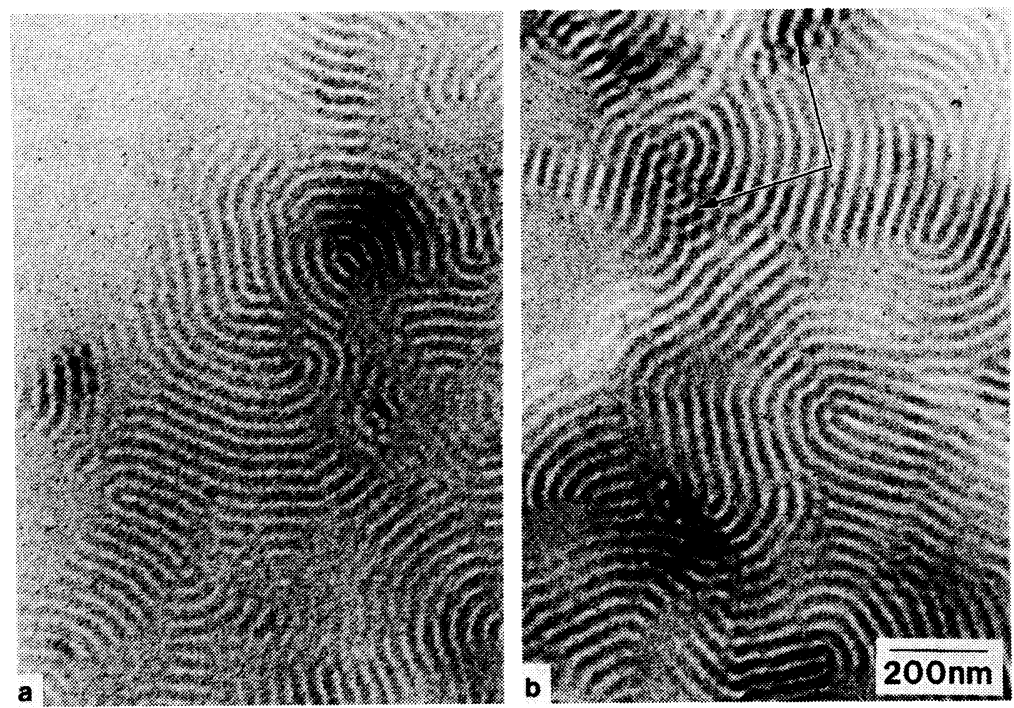

Figure 23. Growth of F414 lamellae annealed at $212^{\circ} \mathrm{C}$ for $15 \mathrm{~min}$ (a) and $45 \mathrm{~min}$ (b). The lamellar patterns in (a) become much more complex as $\tau$ increases in (b). Zipper patterns (shown with arrows) signify regions where lamellae did not connect in sequence. Small black dots are due to platinum decoration.

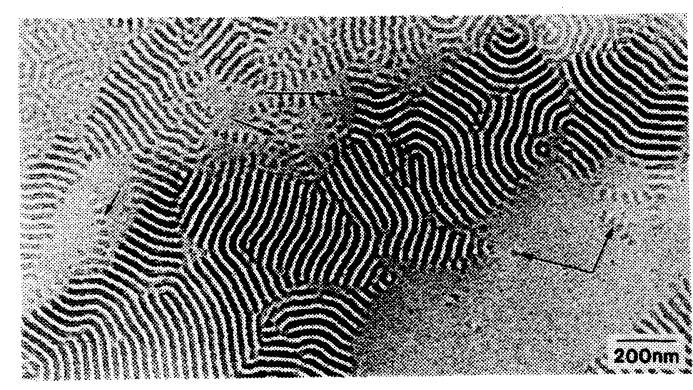

Figure 24. In regions of firmly-established lamellae in F414, structural development occurs by both lamellar growth (as in Figure 23) and cylindrical-domain coalescence (shown by arrows) when the film is heated to $324^{\circ} \mathrm{C}$ for $60 \mathrm{~min}$. This indicates that some aspects of phase separation are due to coarsening. As in Figure 23, the black dots correspond to sputtered platinum.

( $\tau=45 \mathrm{~min})$. Arrows in Figure $23 \mathrm{~b}$ indicate zipper junctions, discontinuities in the lamellae, where lamellae formed from two adjacent regions and attempted to connect. Newly formed lamellae, similar to those in Figure 22b, are seen when F414 was heated for $60 \mathrm{~min}$ at $324^{\circ} \mathrm{C}$ (Figure 24). In addition to new lamellar structure, the presence of two-dimensionally circular domains (the cross-section of cylinders extending into the film) is evident.

Coexistence of nominally cylindrical microstructures and lamellae suggests that, with the lamellae being the equilibrium morphology, some lamellae are formed by cylinders interconnecting. This concept, analogous to coarsening (nucleation) in phase separation, follows as the next logical step from previous work, ${ }^{2}$ in which we provided evidence that some cylindrical microstructures evolved from spherical domains in ultrathin F411 films. However, it is important to realize that cylindrical domains were only found within the proximity of well-formed lamellae; that is, cylindrical domains were not present in areas exhibiting homogeneity.

In areas where no initial phase separation occurred whatsoever, structural formation at $324^{\circ} \mathrm{C}(65 \mathrm{~min})$ was quite different than that reported above. In Figure 25, we see the onset of phase separation as parallel structure "bands," each highlighted by dark lines. Each of these bands measures approximately $110 \mathrm{~nm}$ in thickness (except one which is 180 $\mathrm{nm}$ thick) and is separated by $280 \mathrm{~nm}$. Two cat- 


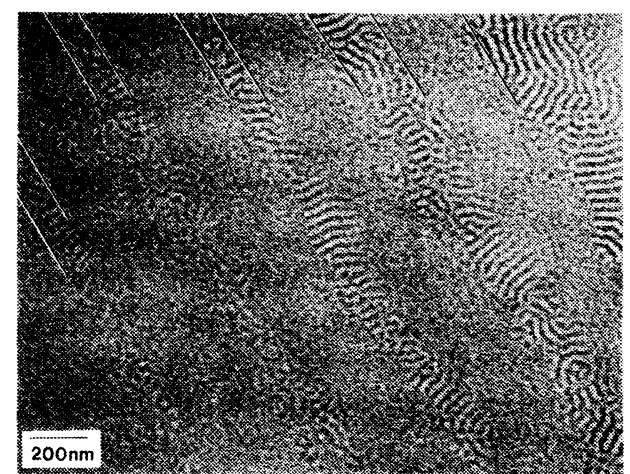

Figure 25. Highlighted by diagonal lines, structure bands are observed forming in homogeneous regions of the $\mathrm{F} 414$ copolymer at $324^{\circ} \mathrm{C}(\tau=65 \mathrm{~min})$. The bands are $110 \mathrm{~nm}$ in thickness (except for one being $180 \mathrm{~nm}$ ) and are equally spaced at $280 \mathrm{~nm}$. Possible explanations for this behavior include flow and phase instabilities (see the text).

egories of instability may be responsible for the bands: flow instability and phase instability.

One type of flow instability is convective motion resulting from nonuniform heating in the film and is referred to as the Benard effect. ${ }^{48,49}$ In this case, anisotropic cells develop in the film; however, the size scale of these cells $(\approx 110 \mathrm{~nm})$ is expected to be on the size scale of the film thickness $(\approx 35 \mathrm{~nm})$ if convection is the mechanism by which they formed. Clearly, the structure bands are much larger (about three times) than the film thickness, suggesting that the Benard effect is not responsible for the observed bands. Another type of flow instability, the Marangoni effect $^{50,51}$ occurs in two-phase systems due to surface tension gradients at a gas-liquid (or melt) interface. However, the copolymer film is coated with carbon, thereby isolating it from the surrounding environment (essentially vacuum) in the microscope. Thus, vacuum-film interactions are minimal, if existent at all, thereby suggesting that this instability cannot be responsible for the structure bands.

The other category of instability that might

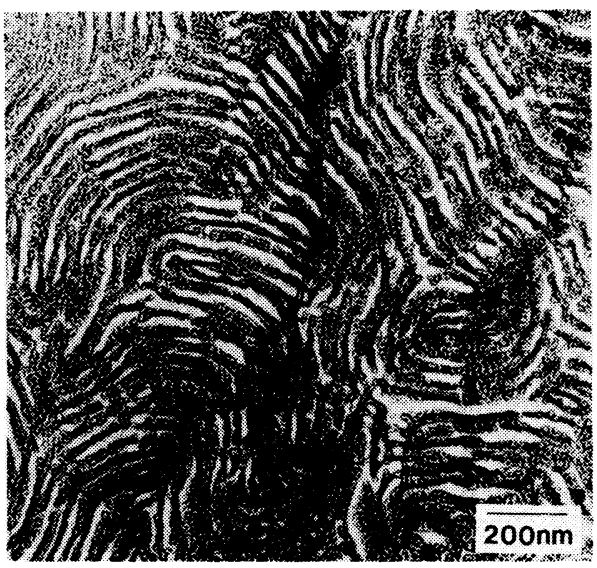

Figure 26. Thermal decomposition in the F414 film, annealed for $45 \mathrm{~min}$ at $400^{\circ} \mathrm{C}$. Granularity of the stained PB matrix suggests that the PB is degrading at this elevated temperature.

be responsible for these bands is phase instability through spinodal decomposition. This mode of phase separation, according to the theory of Cahn and Hilliard, ${ }^{52}$ commences with compositional instability in the free energy of a system and normally results in minor composition variations between phases throughout the entire material. As time progresses, though, the amplitude of the composition difference between phases increases until phase separation is complete. Using a Cahn-Hilliard approach to model phase separation in noncrystalline polymeric systems, Caneba and Soong ${ }^{53}$ predicted that the width of a structure band forming at the onset of spinodal decomposition was $104 \mathrm{~nm}$, later enlarging to $118 \mathrm{~nm}$ upon coarsening. Here, keeping in mind that the modeled system (an anisotropic membrane phase-separating because of nonuniform heating) is different ${ }^{* 3}$ from the annealed F414 SBS copolymer, we see that the theoretical width of a structure band induced by spinodal decomposition is on the same size scale as that observed in Figure 25.

One other viable explanation for the struc-

*3 The membrane was composed of atactic poly(methyl methacrylate) homopolymer with a molecular weight greater than $100000 \mathrm{~g} \mathrm{~mol}^{-1} \cdot{ }^{54}$ 
ture bands (Figure 25) is that they represent the beginning of thermal decomposition, analogous to that found in Figure 19. Heating $\mathrm{F} 414$ to $400^{\circ} \mathrm{C}$ for $45 \mathrm{~min}$ yields the lamellae presented in Figure 26. The stained PB, normally a continuous phase, appears somewhat "granular." This indicates that (a) the PB phase, not the PS phase, is decomposing at this elevated temperature and (b) the structure bands in Figure 25 are not the result of thermal decomposition.

\section{CONCLUSIONS}

The thermal response of microstructures in one Silm multiblock copolymer and in three SBS copolymers has been investigated using dynamic TEM. In the former, partially cured MT3 films exhibited few of the siloxane domains, measuring $2-6 \mathrm{~nm}$ and $16 \mathrm{~nm}$ in diameter, observed in fully cured films. However, in-situ curing at $100^{\circ} \mathrm{C}$ resulted in the appearance of discrete microstructures for heating periods up to $90 \mathrm{~min}$. As the temperature was elevated to $212^{\circ} \mathrm{C}$, the domains increased in number significantly and appeared to resemble the domains found in the fully cured films. Upon heating of the MT3 copolymer to $324^{\circ} \mathrm{C}$, the domains began to coalesce in areas and exhibited signs of grain boundaries.

Several possible explanations have been offered to explain this observed behavior: (1) the film is contracting due to solvent evaporation during the curing; (2) the film is degrading from exposure to the electron beam; or (3) the siloxane microstructural network is decomposing and is accompanied by decreased crystallinity in the imide block at this elevated temperature. The latter explanation, in agreement with diffraction patterns taken at $324^{\circ} \mathrm{C}$, indicates that the matrix exhibits less structure and behaves as a softened rubber at this temperature, thereby allowing the siloxane blocks to aggregate. This proposed behavior is further supported by the fact that MT3 at $300^{\circ} \mathrm{C}$ responds to DMT as a homogeneous fluid. ${ }^{23}$

The investigation of the in-situ annealing of the SBS block copolymers began with a description of $T_{\mathrm{s}}$ predictions (using the thermodynamic theory developed by Williams and coworkers $^{3,14,15}$ ) for SB/SBS copolymers as functions of molecular weight and bulk composition. In the expression $T_{\mathrm{s}}=k \bar{M}_{n}{ }^{c}$, for instance, values of the exponent $c$ are predicted to be 0.81 for diblocks and 0.80 for triblocks at the high-end of the molecular-weight spectrum. Additionally, $T_{\mathrm{s}}$ is found, both theoretically and experimentally, ${ }^{12}$ to increase with an increase in the hard-block fraction of a copolymer (in the range of $0-50 \mathrm{vol}^{\%}$ of hard block).

One major factor that influenced both $T_{\mathrm{s}}$ and phase separation kinetics in the DTEM studies of the SBS copolymers was the presence of $\mathrm{OsO}_{4}$, used as a preferential PB stain to enhance contrast between phases. By assuming a linear relationship between the freevolume fraction remaining after staining $\left(\Phi_{\mathrm{f}}{ }^{\prime}\right)$ and the fraction of free volume occupied by staining molecules, we related $\Phi_{\mathrm{f}}{ }^{\prime}$ to the ratio of viscosities $\left(\eta_{\mathrm{r}}\right)$, or molecular relaxation times, before and after staining in an effort to predict the effects of stain on the time-scale of phase separation. This simple model demonstrated that $\eta_{\mathrm{r}}$ is very sensitive to $\Phi_{\mathrm{f}}{ }^{\prime}$, indicating that, based on diffusional restrictions alone, the stain could retard the time for phase separation or domain evolution by several orders of magnitude. In addition, since $T_{\mathrm{s}} \propto(\Delta \delta)^{2}$ according to the Leary-HendersonWilliams theory, ${ }^{14,15}$ staining could increase $T_{\mathrm{s}}$ dramatically in the stained copolymers by affecting $\delta_{\mathbf{B}}$ so that $\Delta \delta$ increases.

Results from annealing the high-molecularweight copolymer with cylindrical morphology (F411) indicated that the non-equilibrated domains formed upon initial film casting became ordered (into cylinders) as the films were heated to $212^{\circ} \mathrm{C}$ and $324^{\circ} \mathrm{C}$. An ordering parameter, $\kappa$, defined as the ratio of the number of well-structured domains present in a micro- 
graph to the number of domains that would be present under conditions of hexagonal packing, was designed to present a semiquantitative evaluation of the ordering within the micrographs obtained at various times and temperatures. Little change of $\kappa(\tau)$ occurred at $100^{\circ} \mathrm{C}$, indicating insufficient free volume for diffusional mobility, and at $400^{\circ} \mathrm{C}$, suggesting either the onset of homogeneity or thermal decomposition. At intermediate temperatures $\left(212^{\circ} \mathrm{C}\right.$ and $\left.324^{\circ} \mathrm{C}\right), \kappa(\tau)$ displayed initial increases as a function of time (up to $30 \mathrm{~min}$ at $212^{\circ} \mathrm{C}$ and $45 \mathrm{~min}$ at $324^{\circ} \mathrm{C}$ ) and then oscillated at larger $\tau$. Significantly higher domain ordering in the lower- $M$ F416 copolymer than in F411 upon initial film casting confirmed a report ${ }^{47}$ that non-equilibrium effects increase with increasing molecular weight and polydispersity.

Initial casting conditions of the F414 samples resulted in primarily homogeneous regions with sparse lamellar structure. Upon heating, though, structured regions grew in size, with new lamellae and cylinders coexisting. Such coexistence prompted the idea of domain evolution, in which cylinders connect to form lamellae. In addition, structure formation in regions that were initially homogeneous appeared as reproducible bands averaging $110 \mathrm{~nm}$ in thickness and $280 \mathrm{~nm}$ in inter-band spacing. An instability due to phase separation (e.g., spinodal decomposition) is the most viable reason for the observed bands.

Acknowledgments. The authors would like to thank D. Kelly (Cosden Chemical Co.) and R. Eddelman (M\&T Chemicals Inc.) for the block copolymer samples, D. Davis and the staff of the Electron Microscope Laboratory (University of California, Berkeley) for guidance in TEM operations, $R$. Hall of the Mechanical Technology Division (Lawrence Berkeley Laboratory) for assistance in electronics repair/maintenance, and H. R. Wenk of the Department of Geology and Geophysics (University of California, Berkeley) for the loan of the heating stage. This work was supported by the Director, Office of Energy Research, Office of Basic Energy Sciences, Materials Science Division of the U.S. Department of Energy under Contract No. DE-AC03-76SF00098.

\section{APPENDIX}

In eq 4 , we assume that the fraction of free volume remaining after $\mathrm{OsO}_{4}$ staining $\left(\Phi_{\mathrm{f}}{ }^{\prime}\right)$ varies linearly with the fraction of free volume occupied by the staining molecules $\left(\Phi^{\prime}\right)$ :

$$
\Phi_{\mathrm{f}}{ }^{\prime}(T)=a(T)-b(T) \Phi^{\prime} \Phi_{\mathrm{f}}
$$

where $\Phi_{\mathrm{f}}$ is given as a function of temperature in eq 3. To simplify matters, the temperaturedependence notation will be omitted hereafter. Equation 4 must satisfy two imposed boundary conditions (B.C.'s):

$\begin{array}{lll}\text { (1) } \Phi_{\mathrm{f}}{ }^{\prime}=\Phi_{\mathrm{f}}, & \text { when } \Phi^{\prime}=0 \\ \text { (2) } \Phi_{\mathrm{f}}{ }^{\prime}=\Phi_{\mathrm{g}}, & \text { when } \Phi^{\prime}=1\end{array}$

The first condition simply states that the free volume is unaltered in the absence of any stain. The second B.C. implies that when all of the free volume is occupied by stain, the rubber will behave as a glass, with $\Phi_{\mathrm{g}}=0.025$. Upon satisfying boundary condition (1), we find that $a=\Phi_{\mathrm{f}}$, meaning eq 4 can be rewritten as

$$
\Phi_{\mathrm{f}}{ }^{\prime}=\Phi_{\mathrm{f}}\left(1-b \Phi^{\prime}\right)
$$

Evaluation of condition (2) yields

$$
b=\frac{\Phi_{\mathrm{f}}-\Phi_{\mathrm{g}}}{\Phi_{\mathrm{f}}}
$$

We now relate the free-volume fraction to the viscosity by eq 5 and obtain, in the absence of stain,

$$
\eta=A \exp \left(\frac{B}{\Phi_{\mathrm{f}}}\right)
$$

or, in the presence of stain,

$$
\eta^{\prime}=A \exp \left(\frac{B}{\Phi_{\mathrm{f}}^{\prime}}\right)
$$


In the first case (eq A3), $\eta=\eta(T)$ only; however, in the second, $\eta^{\prime}=\eta^{\prime}\left(T, \Phi^{\prime}\right)$. Combining eq A3 and A4 and taking logarithms of both sides yields

$$
\ln \frac{\eta^{\prime}}{\eta}=B\left[\frac{1}{\Phi_{\mathrm{f}}{ }^{\prime}}-\frac{1}{\Phi_{\mathrm{f}}}\right]
$$

If we now substitute eq A1 into eq A5 and rearrange the quantity within the brackets, we obtain

$$
\ln \frac{\eta^{\prime}}{\eta}=B\left[\frac{b \Phi^{\prime}}{\Phi_{\mathrm{f}}\left(1-b \Phi^{\prime}\right)}\right]
$$

To determine $B$, we utilize the third B.C.

(3) $\eta^{\prime}=\eta_{\mathrm{g}}$, when $\Phi^{\prime}=1$ or $\Phi_{\mathrm{f}}{ }^{\prime}=\Phi_{\mathrm{g}}$

This boundary condition agrees with B.C. (2) -i.e., the properties of the rubber (e.g., its free volume and viscosity) behave like those of a glass when all of the free volume of the rubber is occupied by the staining agent. Substituting eq A2 and B.C. (3) into eq A6 yields

$$
B=\left[\frac{\Phi_{\mathrm{f}} \Phi_{\mathrm{g}}}{\Phi_{\mathrm{f}}-\Phi_{\mathrm{g}}}\right] \ln \frac{\eta_{\mathrm{g}}}{\eta}
$$

The result, for $\Phi^{\prime}$-dependence of the stainedsample viscosity, is

$$
\ln \frac{\eta^{\prime}\left(\Phi^{\prime}\right)}{\eta}=\left(\ln \frac{\eta_{\mathrm{g}}}{\eta}\right)\left[\frac{\Phi_{\mathrm{f}} \Phi^{\prime}}{\Phi_{\mathrm{f}}\left(1-\Phi^{\prime}\right)+\Phi_{\mathrm{g}} \Phi^{\prime}}\right]
$$

The viscosity ratio $\left(\ln \eta_{\mathrm{g}} / \eta\right)$ can be evaluated from the WLF equation: ${ }^{39}$

$$
\ln \frac{\eta_{\mathrm{g}}}{\eta}=\frac{D_{1}\left(T-T_{\mathrm{g}}\right)}{D_{2}+\left(T-T_{\mathrm{g}}\right)}
$$

where $D_{1}=40.16$ and $D_{2}=51.6$ degrees Kelvin. The glass-transition temperature $\left(T_{\mathrm{g}}\right)$ for the PB is taken to be $193.15 \mathrm{~K}$. The use of eq $A 8$ and eq 4 allows us to obtain the $\eta_{\mathrm{r}} v s$. $\Phi_{\mathrm{f}}{ }^{\prime}$ plot in Figure 14, given parameters $\Phi_{\mathrm{f}}(T)$ and $B(T)$ displayed in Table II.

\section{REFERENCES}

1. R. J. Spontak, M. C. Williams, and C. N. Schooley,
J. Mater. Sci., 21, 3173 (1986).

2. R. J. Spontak, M. C. Williams, and D. A. Agard, Polymer, 29, 387 (1988).

3. R. J. Spontak, M. C. Williams, and D. A. Agard, Macromolecules, 21, 1377 (1988).

4. G. Kraus and T. Hashimoto, J. Appl. Polym. Sci., 27, 1745 (1982).

5. R. Roe, M. Fishkis, and J. C. Chang, Macromolecules, 14, 1091 (1981).

6. T. Hashimoto, K. Kowsaka, M. Shibayama, and S. Suehiro, Macromolecules, 19, 750 (1986).

7. T. Hashimoto, M. Shibayama, and H. Kawai, Macromolecules, 16, 1093 (1983).

8. F. S. Bates and M. A. Hartney, Macromolecules, 18, 2478 (1985).

9. R. W. Richards and J. L. Thomason, Polymer, 24, 1089 (1983).

10. R. W. Richards and J. L. Thomason, Macromolecules, 16, 982 (1983).

11. D. F. Leary and M. C. Williams, J. Polym. Sci., Polym. Phys. Ed., 12, 265 (1974).

12. L. M. Leung and J. T. Koberstein, Macromolecules, 19, 706 (1986).

13. C. D. Han and J. Kim, J. Polym. Sci., Polym. Phys. Ed., 25, 1741 (1987).

14. D. F. Leary and M. C. Williams, J. Polym. Sci., Polym. Phys. Ed., 11, 345 (1973).

15. C. P. Henderson and M. C. Williams, J. Polym. Sci., Polym. Phys. Ed., 23, 1001 (1985).

16. L. Leibler, Macromolecules, 13, 1602 (1980).

17. G. H. Fredrickson and R. G. Larson, J. Chem. Phys., 86, 1553 (1987).

18. R. G. Larson and G. H. Fredrickson, Macromolecules, 20, 1897 (1987).

19. T. Hashimoto, Macromolecules, 20, 465 (1987).

20. A. Berger, in "Polyimides: Synthesis, Characterization, and Applications," Vol. I, K. L. Mittal, Ed., Plenum Press, New York, 1984, pp 67-75.

21. A. Berger, U. S. Patent 4,139,547 (1979).

22. A. Berger, U. S. Patent 4,395,527 (1983).

23. R. J. Spontak and M. C. Williams, J. Appl. Polym. Sci., submitted.

24. R. Eddelman, $M$ \& $\mathrm{T}$ Chemicals, Inc., personal communication, 1987.

25. M \& T 3500 Siloxane Polyimide Data Sheet, $M \&$ T Chemicals, Rahway, New Jersey, 1983.

26. R. J. Spontak, in Proceedings of the 44th Annual Meeting of the Electron Microscopy Society of America, G. W. Bailey, Ed. San Francisco Press Inc., San Francisco, 1986, pp 788-789.

27. T. Hashimoto, K. Kowsaka, M. Shibayama, and H. Kawai, Macromolecules, 19, 754 (1986).

28. E. R. Pico and M. C. Williams, J. Polym. Sci., Polym. Phys. Ed., 15, 1585 (1977).

29. L. C. Sawyer and D. T. Grubb, "Polymer Microscopy,"Chapman and Hall Ltd., London, 1987, p 77. 
30. F. Annighofer and W. Gronski, Makromol. Chem., 185, 2213 (1984).

31. G. C. Davis, B. A. Heath, and G. Gildenblat, in "Polyimides: Synthesis, Characterization, and Applications." Vol. II, K. L. Mittal, Ed., Plenum Press, New York, 1984, pp 847-869.

32. J. J. King and B. H. Lee, in "High Performance Polymers: Their Origin and Development," R. B. Seymour and G. S. Kirshenbaum, Ed., Elsevier Science Publishing Co., Inc., New York, 1986, pp 317-330.

33. E. P. Butler and K. F. Hale, in "Practical Methods in Electron Microscopy," Vol. 9, A. M. Glauert, Ed., North-Holland Publishing Co., Amsterdam, 1981.

34. G. Hadziioannou and A. Skoulios, Macromolecules, 15, 267 (1982).

35. T. E. Schichtel and K. Binder, Macromolecules, 20, 1671 (1987).

36. R. J. Spontak and M. C. Williams, J. Polym. Sci., Polym. Phys. Ed., submitted.

37. R. J. Spontak, PhD. Thesis, University of California, Berkeley, 1988.

38. B. Endres, R. W. Garbella, and J. H. Wendorff, Colloid \& Polym. Sci., 263, 361 (1985).

39. D. W. van Krevelen, "Properties of Polymers: Their Estimation and Correlation with Chemical Structure," Elsevier Scientific Publishing Company, Amsterdam, 1976, pp 339-341.
40. G. S. Whitby, C. C. Davis, and R. F. Dunbrook, "Synthetic Rubber," John Wiley \& Sons, Inc., New York, 1954, p 350.

41. A. Baumgartner and D. W. Heermann, Polymer, 27, 1777 (1986).

42. S. Reich and Y. Cohen, J. Polym. Sci., Polym. Phys, Ed., 19, 1255 (1981).

43. Y. S. Lipatov, Colloid \& Polym. Sci., 264, 377 (1986).

44. W. H. Beeer, Ed., "CRC Handbook of Mathematical Sciences," 5th ed. CRC Press, Inc., Boca Raton, Florida, 1978, p 191.

45. M. Xie and Y. Camberlin, Makromol. Chem., 187, 383 (1986).

46. R. N. Haward, Ed., "The Physics of Glassy Polymers," Applied Science Publishers Ltd., London, 1973, pp 460-466.

47. D. J. Kinning and E. L. Thomas, Macromolecules, 17, 1712 (1984).

48. W. R. Krigbaum, S. Yazgan, and W. R. Tolbert, $J$. Polym. Sci., Polym. Phys Ed., 11, 511 (1973).

49. J. R. A. Pearson, J. Fluid Mech., 4, 489 (1958).

50. C. H. Chun, Acta Astronautica, 7, 479 (1980).

51. L. G. Napolitano, Acta Astronautica, 9, 199 (1982).

52. J. W. Cahn and J. E. Hilliard, J. Chem. Phys., 28, 258 (1958).

53. G. T. Caneba and D. S. Soong, Macromolecules, 18, 2545 (1985).

54. G. T. Caneba and D. S. Soong, Macromolecules, 18 , 2538 (1985). 